\title{
The Electric Vehicle Routing Problem with Time Windows and Synchronised Mobile Battery Swapping
}

\author{
Ramin Raeesi \\ Centre for Logistics and Heuristic Optimisation (CLHO), Kent Business School, University of Kent, Chatham
} ME44TE,UK,r.raeesi@kent.ac.uk

Konstantinos G. Zografos

Centre for Transport and Logistics (CENTRAL), Department of Management Science, Lancaster University Management School, Lancaster LA1 4YX, UK, k.zografos@lancaster.ac.uk 


\begin{abstract}
This paper introduces an alternative to intra-route recharging of Electric Commercial Vehicles (ECVs) used for freight distribution by exploiting new pertinent technological developments that make mobile battery swapping possible. The Electric Vehicle Routing Problem with Time Windows and Synchronised Mobile Battery Swapping (EVRPTW-SMBS) is introduced in which route planning is carried out in two interdependent levels: (i) for the ECVs to deliver customers' demands, and (ii) for the Battery Swapping Vans (BSVs) to swap the depleted battery on an ECV with a fully charged one at a designated time and space. Each BSV route can provide the battery swapping service to multiple ECVs, and each ECV can extend its autonomy by requesting the battery swapping service for as many times as required with no need to divert from its original delivery route. The EVRPTW-SMBS opens up multiple opportunities to facilitate eco-friendly goods distribution using ECVs and brings in extra flexibility and cost savings. At the same time, it is a challenging problem to tackle mainly due to the interdependence problem that stems from the spatio-temporal synchronisation requirement between the vehicles in the two levels (i.e. ECVs and BSVs). To tackle these complications, the paper proposes a methodology for exact evaluation of an EVRPTW-SMBS solution based on a two-stage hybridisation of a dynamic programming and an integer programming algorithm, and places the resulting procedure at the heart of an intensified large neighbourhood search algorithm to solve instances of the EVRPTW-SMBS efficiently. A library of EVRPTW-SMBS test instances is developed and used to demonstrate the added value of the proposed problem variant and the efficiency of the proposed algorithms. Our results demonstrate the benefits of using BSVs in the design of the delivery routes for ECVs, and indicate that a particular variant of the proposed algorithms which is based on a specific lexicographical decomposition routine can efficiently approximate the optimal solution to the EVRPTW-SMBS.
\end{abstract}

Keywords: Electric vehicle, routing and scheduling, recharging, battery swapping, spatio-temporal synchronisation 


\section{Introduction}

Urban Freight Distribution (UFD) plays a pivotal role in the transportation and delivery of the goods required to sustain more than half of the world's population that now live in urban areas (Ritchie and Roser, 2018). However, it is generating significant externalities such as traffic congestion and environmental pollution. Freight vehicles typically represent $8 \%$ to $15 \%$ of total traffic flow in urban areas (MDS Transmodal, 2012) and are responsible for $25 \%$ of urban transport related $\mathrm{CO}_{2}$ emissions and $30 \%$ to $50 \%$ of other transport related pollutants (e.g. Particulate Matter and Nitrogen Oxide) (Alice/Ertrac, 2015). This large and ever-increasing level of emissions from urban freight transport activities has attracted the attention of policy makers and national governments. The European Commission has, for instance, set a target for "essentially $\mathrm{CO}_{2}$-free city logistics in urban centres by 2030" (European Commission, 2011). Meeting such target would inevitably entail facilitating the conversion of conventionally fuelled UFD fleets into Electric Commercial Vehicles (ECVs) with zero local emissions. This conversion of the fleet, however, is constrained by ECVs' reduced driving range, long recharging time, and unevenly scattered Charging Stations (CSs). While reports (Committee on Climate Change, 2010) suggest electric light goods vehicles will be cost-saving compared to conventionally fuelled vehicles by 2030 (Allen et al., 2017), for a smooth transition phase, short term operational solutions are crucial.

The Electric Vehicle Routing Problem with Time-Windows (EVRPTW) (Schneider et al., 2014) is a variant of the VRP that aims at aiding companies operating ECVs to overcome "range anxiety" by developing solutions that comprise detours to available CSs for intra-route recharging. The primary challenge in addressing the EVRPTWs, that distinguishes them from their Green VRP (G-VRP) (Erdogan \& Miller-Hooks, 2012) counterpart, is in the significantly larger recharging time required to refill ECVs' batteries as compared to other alternative fuel vehicles. This limitation has implications mainly with regards to meeting customers' time-windows, and thus in the presence of realistic time windows the solution yielded could be too expensive in terms of the number of ECVs required and the total distance travelled.

Despite the ongoing expansion of the electric vehicles CSs network and the advancement of rapid recharging technologies, there are still several practical questions regarding intra-route refuelling as a way to address the limited autonomy of ECVs for freight distribution. In particular, one key question that often arises in this area is related to the "CS ownership" (Montoya et al. 2017). On the one hand, public CSs are not always allowed to be used by logistics companies (Worley et al. 2012). For example, Tesla's Supercharger Fair Use Policy ${ }^{1}$ dictates that vehicles used for commercial purposes are expected not to use the public Supercharger network (a network of 1,317 Supercharger stations) to keep it available for non-commercial users. Even if this is not an issue and public CSs are open to commercial users, a major concern is the uncertainty tied to the availability of a CS upon the arrival of the ECV (Sweda et al. 2017, Kullman et al. 2018, Montoya et al. 2017, Froger et al. 2019). A public CS occupied by another public or commercial vehicle upon the arrival of the ECV may imply a considerably large queuing time for the service point to become available again, and can thus disrupt the pre-determined routing plan and jeopardise the fulfilment of delivery tasks. Therefore, the use of public CSs to charge ECVs in a routing context has received little attention (Sweda et al. 2015, Kullman et al. 2016) and the majority of the EVRP literature assumes implicitly that the charging infrastructure is privately owned by the ECV operator (Froger et al. 2019).

On the other hand, what has been largely ignored thus far in EVRPs with privately owned CSs corresponds to the large set-up cost of CSs and the location allocation aspects of the problem. Opening and maintaining a CS can be significantly costly (cost of required land and infrastructure, energy supply, operating and maintenance cost) and the tradeoff between acquiring more ECVs to operate shorter routes, and establishing a CS is yet unknown and very much problem dependent. These arguments regarding the ownership of CSs apply also to the case of Battery Swapping Stations (BSSs); while battery swapping at a BSS takes a much shorter time than recharging the battery, the use of BSSs in the routing of ECVs with intra-route swapping (instead of recharging), as proposed in Yang and Sun (2015) and Hof et al. (2017), brings about the same complications as the EVRPs with CSs do, and ignoring these real life considerations can hinder companies from adopting these solutions.

To address the aforementioned limitations, in this study we introduce a paradigm shift in goods distribution using electric vehicles by exploiting new relevant technological developments that make mobile battery swapping possible. The development of a new fast battery swapping device installed on a Battery Swapping Van (BSV), documented in patents (Gao et al., 2012) and (Lu and Zhou, 2013), and justified and corroborated by the study of Shao et al. (2017), opens up new possibilities to logistics by providing a 'non-stationary' battery swapping mode, as opposed to the 'stationary' battery

${ }^{1}$ https://www.tesla.com/en_GB/about/legal?\#supercharger-fair-use 
swapping mode as in BSS-LRP. Hence, in this study we introduce and formulate a new class of the EVRPTWs called the Electric Vehicle Routing Problem with Time Windows and Synchronised Mobile Battery Swapping (EVRPTW-SMBS) that is motivated by the aforementioned technological development. The underlying idea in the EVRPTW-SMBS is to swap the depleted battery on an ECV with a fully-charged one using a BSV at a designated time and space, without the need to divert the delivery route of the ECV. Therefore, in the proposed problem variant routing must be carried out in two synchronised levels for the ECVs which carry out the delivery tasks and for the BSVs which are responsible for extending the autonomy of the running ECVs on-the-fly. The logistic model proposed in the EVRPTW-SMBS brings about extra flexibility in goods distribution using ECVs, and has a potential to significantly reduce costs corresponding to the assignment of BSVs and ECVs to delivery routes when compared with costs incurred by privately owning CSs and diverting the route of ECVs to them for intra-route recharging.

The EVRPTW-SMBS is a very difficult problem to solve due to the spatio-temporal synchronisation requirements and the existence of the 'interdependence' between the routes of the two levels. As the proposed mathematical formulation for the problem is only able to address instances of a limited size, the paper proposes also different variants of a heuristic solution algorithm corresponding to a Dynamic Programming based Intensified Large Neighbourhood Search (DP-ILNS) algorithm to find near optimal solutions to practically sized instances of the problem within a reasonable computational time.

The contribution of this paper is multi-fold: (i) the use of mobile battery swapping in electric vehicle routing problems is proposed for the first time as an alternative to solutions based on intra-route recharging, (ii) the EVRPTW-SMBS is introduced and formulated as a Mixed Integer Linear Programming (MILP) model with spatio-temporal synchronisation constraints that ensure a BSV and an ECV will be present at a designated time and location to perform a planned battery swap, (iii) a two-stage procedure based on the combination of a dynamic programming algorithm and a label-selecting integer programming model is proposed for exact evaluation of an EVRPTW-SMBS solution and to tackle the interdependence problem, and (iv) three variants of a tailored heuristic solution algorithm based on different lexicographic decomposition strategies are proposed for the problem.

In the remainder of the paper, in section 2, a survey on the most pertinent literature is presented. Section 3 of the paper describes the EVRPTW-SMBS. Section 4 develops the solution algorithm for the problem. Section 5 presents the computational results; and finally, section 6 is the discussion and concluding remarks section.

\section{Previous related work}

The significant share of road freight distribution in the global emissions of Greenhouse Gases (GHGs) and other environmental pollutants has motivated a surge of interest in the study of Vehicle Routing and Scheduling Problems (VRSPs) with environmental considerations in recent years. Research work in this area might be broadly categorised into two streams: (i) the Emissions minimising VRPs (EM-VRPs) comprising the pollution-routing problem (Bektas \& Laporte, 2011) and its variants ( Franceschetti et al., 2013; Demir et al., 2014; Koc et al., 2014; Androutsopoulos \& Zografos, 2017; Raeesi \& Zografos, 2019), that aim at minimising the fuel consumption incurred by the delivery routes as a proxy for emissions, and (ii) the Green VRPs (G-VRPs) that are concerned with routing a fleet of vehicles that run on a cleaner alternative fuel (Erdogan \& Miller-Hooks, 2012; Raeesi \& O'Sullivan, 2014; Salimifard \& Raeesi, 2014) or electric batteries (Conrad \& Figliozzi, 2011; Bruglieri et al., 2015; Schneider et al., 2014; Desaulniers et al., 2016; Hiermann et al., 2016). There is also recent research that bridges these two categories by routing a mixed fleet of electric and conventional vehicles (Goeke \& Schneider, 2015; Macrina et al., 2019). The interested reader is referred to Bektas et al. (2019) for a recent review of the key papers in the field. We also refer the reader to the paper by Pelletier et al. (2016) on goods distribution with ECVs that serves as a good starting point to discover the fundamentals of the ECV technology and its relevant economic and operational aspects. While the use of mobile battery swapping in designing delivery routes for ECVs and the problem proposed in this paper has not been previously studied, in what follows, we present a concise review of the papers in the area of EVRPTW, and a brief discussion on two classes of routing problems that may not be explicitly related to this study but share some key features with the problem considered in this paper.

The EVRPTW (Schneider et al., 2014) can be viewed as a special case of the G-VRP (Erdogan \& Miller-Hooks, 2012) where capacity constraints and time-windows are added to the problem, and significantly larger refuelling (recharging) time is assumed. In the variant considered by Schneider et al. (2014) a minimum number of ECVs must be assigned to energy-feasible delivery routes (potentially visiting one or several CSs) that visit each customer exactly once 
during their pre-defined time-windows, such that the total capacity constraint of the ECVs is not violated and the total distance travelled is minimised. Due to the limited driving range of ECVs, the core complication in the EVRPTW is related to the introduction of minimal detours in the vehicle routes to visit available CSs on the working graph to fully recharge their battery and carry out the delivery task. Schneider et al. (2014) develop an algorithm based on the hybridisation of a Variable Neighbourhood Search (VNS) algorithm with a Tabu Search (TS) heuristic to address the proposed variant.

To allow more flexibility in the design of the ECV delivery routes, Keskin and Çatay (2016) relax the full recharging restriction and allow partial recharging at a CS. An Adaptive Large Neighbourhood Search (ALNS) algorithm that comprises new heuristics for station removal and station insertion is employed to solve the proposed problem. Their computational results demonstrate that with partial recharging instead of full recharging, the solution to a few test instances can be improved.

The use of different charging technologies has also been considered in several studies; Felipe et al. (2014) propose a heuristic to solve a variant in which in addition to the decision on the charging level at a CS, the technology used for recharging e.g. regular or fast recharging is considered. Montoya et al. (2017) discuss several real-life considerations in EVRPs in relation to ECVs energy consumption, the charging infrastructure ownership, the capacity of the CSs and the battery charging process. In particular, they argue that the recharging level of the battery is a non-linear function of the recharging time and study the EVRP with a nonlinear recharging function. They propose a hybrid metaheuristic for the problem and show that neglecting nonlinear charging may lead to infeasible or overly expensive solutions.

Hiermann et al. (2016) consider the fleet size and mix in the EVRPTWs where the available vehicle types in the fleet differ in terms of their capacity, battery size and acquisition cost. Goeke and Schneider (2015) study the EVRPTW with a mixed fleet of ECVs and conventional internal combustion commercial vehicles. A distinctive feature of their study is that instead of simply assuming energy consumption is a linear function of the distance travelled, they utilise an energy consumption model that takes speed, road slope and vehicle payload into account. In the same vein, Basso et al. (2019) incorporate into the routing decision an improved and more accurate energy consumption estimation model comprising detailed topography and speed profiles.

As was discussed earlier, and as it is argued in Forger et al. (2019), the majority of the EVRP literature assumes implicitly that the charging infrastructure is privately owned by the ECV operator. However, in none of the studies discussed above is the cost of CSs taken into account explicitly. There is, however, a separate stream of research that focuses on location-routing models where vehicle routing and the siting of CSs (or battery swapping stations) is simultaneously considered (Worley et al. 2012, Yang \& Sun, 2015, Schiffer \& Walther, 2017, Hof et al. 2017). Schiffer and Walther (2017) study the electric location routing problem with time windows and partial recharging and consider simultaneously the routing of electric vehicles and the siting decisions for CSs with different recharging technologies. Yang and Sun (2015) consider the use of BSSs in EVRPs and propose the electric vehicles battery swap stations location routing problem, which aims to determine the location of BSSs and the routes of ECVs simultaneously.

In this paper, we employ a completely different approach from the literature that has thus far only considered 'stationary' recharging or battery swapping technologies in the design of delivery routes for ECVs. We propose to use mobile battery swapping vans to service ECVs on-the-fly without any need to divert their routes. While this has not been previously studied, due to the existence of the spatio-temporal synchronisation requirement of an ECV with a BSV in the proposed model, there are some similarities with two classes of routing problems corresponding to: (i) the two-Echelon VRP with Satellite Synchronisation (2E-VRP-SS) and (ii) the Coordinated Truck-Drone Routing Problem (CTDRP).

In 2E-VRP-SSs (Anderluh et al., 2017; Crainic et al., 2009; Grangier et al., 2016) two distinct fleets of vehicles are used to make deliveries. First-level vehicles pick up requests at a distribution centre and bring them to intermediate sites (called satellites) where the requests are transferred to second-level vehicles for ultimate delivery. The required temporal synchronisation of the vehicles in the first echelon with the vehicles of the second echelon at a satellite, therefore, resembles to some extent the kind of synchronisation one must establish for a planned battery swap in EVRPTW-SMBS. In CTDRPs (Murray and Chu, 2015; Poikonen et al., 2017; Carlsson and Song, 2018; Karak and Abdelghany, 2019; Schermer et al., 2019; González-Rodríguez et al., 2020; Kitjacharoenchai et al., 2020) trucks and drones work in tandem to deliver customers' demands. Trucks serve as a mobile platform for battery swapping, reloading packages for delivery and launching drones to make the deliveries. Thus, the role of a truck in the CTDRPs resembles the role of a BSV in the EVRPTW-SMBS, and similar to the EMVRPTW-SMBS, there is a need to determine rendezvous points where batteries used by the drones are replaced by fully-charged ones. 
The main complication that arises in establishing such synchronisations is due to the fact that unlike in the standard VRPs where vehicles are independent of one another, in VRPs with synchronisation constraints a change in one route may have effects on other routes, and in the worst case, a change in one route may render all other routes infeasible. This problem is known as the 'interdependence problem' (Drexl, 2012) and is the key challenge in solution development for 2E-VRP-SSs and CTDRPs. Grangier et al. (2016) propose to represent the time constraints in the 2E-VRP-SS as a directed acyclic graph called a 'precedence graph'. They use this graph in their route scheduling and feasibility algorithm which is placed at the centre of their proposed ALNS algorithm for the problem. Anderluh et al. (2017) consider synchronisation between cargo bikes and vans, and propose a heuristic based on a greedy randomized adaptive search procedure with path relinking for the problem. González-Rodríguez et al. (2020) consider the truck-drone team logistics problem and propose an iterated greedy heuristic based on the iterative process of destruction and reconstruction of solutions. Each solution in their algorithm is coded as a resource-type vector to track if a node is visited by the drone, by the truck or by both the drone and the truck. Karak and Abdelghany (2019) use an extension of the classic Clarke and Wright algorithm to address the hybrid vehicle-drone routing problem for pick-up and delivery services. Kitjacharoenchai et al. (2020) propose a drone truck route construction heuristic and a LNS for a synchronised truck-drone operation in which multiple drones are allowed to fly from a truck, serve one or multiple customers, and return to the same truck for a battery swap and package retrieval.

Despite the aforementioned similarity, there are also key differences between the EVRPTW-SMBS and the two variants discussed above. Compared with the 2E-VRP-SS, the EVRPTW-SMBS is a harder problem to address because, in addition to the temporal synchronisation requirement in the 2E-VRP-SS, there is also a need to establish spatial synchronisation between the vehicles in the two levels in EVRPTW-SMBS. That is, unlike in the 2E-VRP-SS where the location of the satellites are known in advance, in the context of the EVRPTW-SMBS the designated location of battery swapping is only an outcome of optimisation and is not known a priori. Hence, the interdependence problem is much more strongly present in EVRPTW-SMBS and the routes in each of the two levels might be significantly affected by any slight change in the routes of the other level. As regards the CTDRPs, spatio-temporal synchronisation requirements are involved as they are in the case of the EVRPTW-SMBS, but they are usually easier to handle as in the dominant variant of the CTDRP only one truck is considered. There is a more similar variant of the CTDRP in which multiple trucks and multiple drones are considered (Poikonen et al., 2017; Schermer et al., 2019), but in this variant it is usually assumed that a drone returns to the same truck from which it is launched, and it can visit one customer only on each route. Moreover, no timewindows on customers are usually considered in this variant and drones and trucks can wait indefinitely for one another at the rendezvous points. In the EVRPTW-SMBS, however, ECVs can request service from different BSVs, and ECVs services cannot be delayed. Therefore, solution algorithms proposed for these problem variants are not directly applicable to the EVRPTW-SMBS and there is a need to develop a dedicated solution methodology for the problem. In this paper, we propose a DP-ILNS algorithm which is tailored for the EVRPTW-SMBS.

\section{The EVRPTW-SMBS: formal description and formulation}

In this section, a formal description of the EVRPTW-SMBS is first provided and the notation and key assumptions adopted by the paper are discussed. Next, a small illustrative example of the problem is presented to establish a case for it, and following that, the mathematical formulation of the problem is given. A list of all key notation used in the paper is provided in appendix A.

\subsection{Formal description of the problem}

The EVRPTW-SMBS is defined on a complete, directed graph $G=(N, A)$, where $N$ is the set of network nodes and $A$ is the set of directed arcs. The set $N=\{D \cup C\}$ is comprised of the $\operatorname{depot} D=\{0, \sigma\}$, with $\{\sigma\}$ being a dummy copy of $\{0\}$ (referred to as the final depot) and customer nodes $C=\{1,2, \ldots, n\}$. Each customer $i \in C$ is associated with a certain demand $q_{i}$ to be delivered within its pre-determined hard time window, denoted by $\left[e_{i}, l_{i}\right]$, with service time $s_{i}$. The depot working hours, which is considered as the planning horizon, is denoted by $T=\left[e_{0}, l_{0}\right]$. The set of directed arcs is defined as $A=\left\{(i, j) \mid i \in N_{0}, j \in N_{\sigma}, i \neq j\right\}$, where $N_{0}=\{0\} \cup C$ and $N_{\sigma}=C \cup\{\sigma\}$. To each $\operatorname{arc}(i, j) \in A$, a distance $d_{i j}$, and a travel time $t_{i j}$ is attributed. There is a fleet of homogeneous ECVs and a fleet of homogenous electric BSVs that are all fully charged and located at the central depot. To each ECV a fixed acquisition $\operatorname{cost} \mathcal{C}_{e}$, a maximum payload $Q_{e}$, a battery capacity $B_{e}$, and an energy consumption rate per unit distance travelled $r_{e}$ is attributed. Each BSV, on the other hand, is an electric van that has a fixed acquisition $\operatorname{cost}$ of $\mathcal{C}_{b}$, can carry a maximum number of batteries $Q_{b}$, has a battery capacity $B_{b}$, 
and an energy consumption rate $r_{b}$. The operational cost of each unit distance travelled by an ECV and a BSV is denoted by $\mathcal{C}_{d_{e}}$ and $\mathcal{C}_{d_{b}}$, resepectively. Moreover, the following key assumptions are made in the proposed EVRPTW-SMBS:

- The tasks of ECVs and BSVs are not interchangeable. That is, ECVs are only employed to deliver the requests of customers, and BSVs are only used when battery swapping is required by an ECV, and they cannot be used for delivery.

- Battery swapping must be carried out at a customer location and realistically it cannot be done simultaneous with the ECV providing service at the customer. Hence, battery swapping can only take place either before (e.g. while the ECV is waiting for customer service to start, if "possible") or right after the ECV service at the customer is over. More precisely, let $y_{i}$ be the arrival time of the ECV at customer $i \in C, a_{i}$ the service start time, $b_{i}$ the ECV departure time after service and battery swapping completion, $\theta_{i}$ the start time of battery swapping service and $s$ the time units required for swapping the battery on the ECV; then $\theta_{i}$ is determined as follows:

$$
\theta_{i}=\left\{\begin{array}{cl}
\mathbb{C} \in\left[y_{i}, e_{i}-s\right], & \text { if } y_{i} \leq e_{i}-s \\
a_{i}+s_{i}, & \text { otherwise }
\end{array}\right.
$$

That is, if the ECV arrives early "enough" (before $e_{i}-s$ ) at the customer location, the BSV must start the battery swapping service at a time over the interval $\left[a_{i}, e_{i}-s\right]$; otherwise the battery swapping service begins immediately upon completion of the customer service. This synchronisation strategy follows the key principle that the customer service must not be delayed due to a battery swapping service; in other words, customer service has priority over the battery swapping service. In Figure 1, different possible scenarios for the arrival time of the ECV at a customer location and the resulting battery swapping start times are illustrated over a timeline. In Figure 1.a battery swapping must take place at any time over the shaded box; however, in all other case, swapping takes place right after the completion of ECV service at the customer. Note that while in the particular case of Figure 1.b it looks possible to start swapping before servicing the customer for an earlier departure time, in a general case when the customer's time window is tight and swapping time is larger than the width of customer's time window, the result of delaying the customer service would be the violation of the later boundary of its time window. Note also that a BSV can arrive at a designated point of swapping before the swapping service can actually start, but it has to wait.

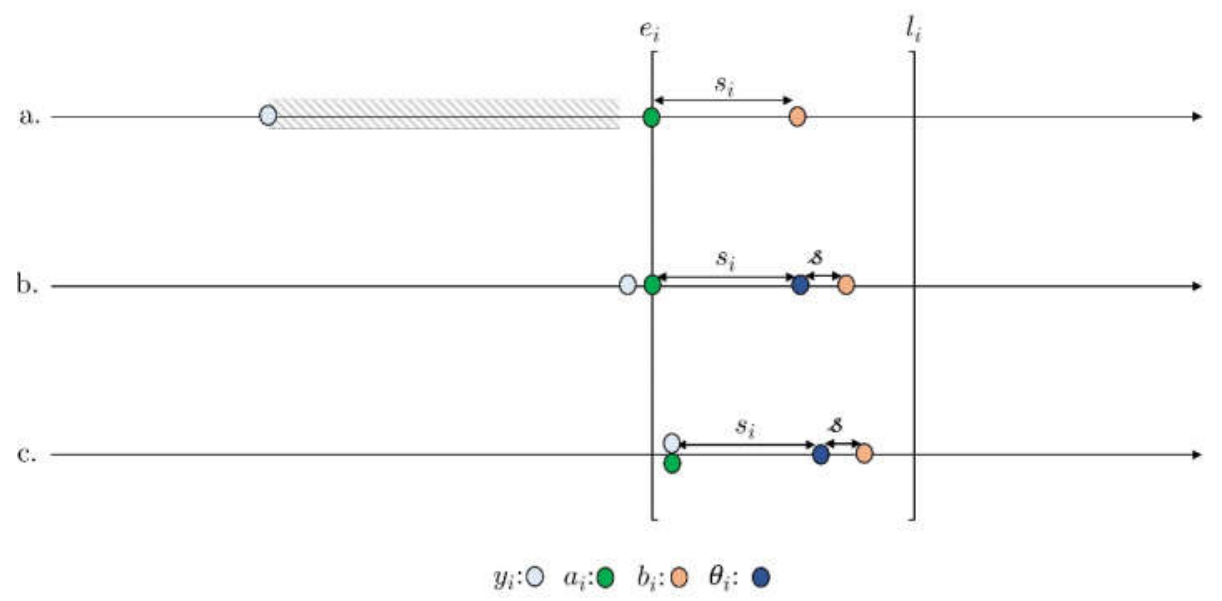

Figure 1 Possible scenarios for the synchronisation of the arrival of the ECV and the BSV

It may be worth noting that confining the allowable battery swapping locations to customer locations is not a restrictive assumption, and it is possible to introduce other separate designated points of swapping to $G$.

- BSVs are not allowed to require a battery swap from other BSVs during their trip.

- An ECV can ask for the battery swapping service for as many times as required during its trip, and there is no restriction for a BSV to serve the same ECV several times.

- The fixed vehicle acquisition costs for ECVs and BSVs comprise the initial purchasing investments, all subsequent operational and maintenance costs, and the inflationary and depreciation factors over a certain lifetime (e.g. a 5-year period). The calculation of the corresponding cost coefficients (i.e. $\mathcal{C}_{e}$ and $\mathcal{C}_{b}$ ) for each time-period (e.g. a day) can be done through the methodologies discussed in relevant studies focusing on the Total Cost of Ownership (TCO) calculations for ECVs in routing contexts (Davis and Figliozzi, 2013; Lee et al., 2013; Feng and Figliozzi, 2013; 
Schiffer et al., 2017). TCO calculations consider costs over time as well as time-dependent discounts and depreciations and comprise the one-time investments and the periodically arising costs. The operational cost of each unit distance travelled by an ECV and a BSV (i.e. $\mathcal{C}_{d_{e}}$ and $\mathcal{C}_{d_{b}}$ ), on the other hand, is calculated through an estimation of distance-dependent maintenance costs, energy cost and driver wage. Also note that since multiple trips are not considered in our model, to execute each route a vehicle must be acquired and therefore the cost of serving an ECV or a BSV route corresponds to the acquisition cost of an ECV or a BSV, respectively.

The aim of the EVRPTW-SMBS is to determine an optimal composition of ECVs and BSVs in the fleet to operate routes that start and finish at the depot, such that every customer is visited exactly once by an ECV within its pre-defined time-window, and the payload and battery capacity of the ECVs and BSVs and the working day limits are not violated. The objective of the EVRPTW-SMBS is to minimise a composite function comprising the cost of the ECVs and BSVs assigned to the routes and the total distance travelled by them.

\subsection{A business case for the use of mobile battery swapping and an illustrative example}

Just like solutions based on intra-route recharging for increasing ECVs autonomy, the competitiveness of solutions based on mobile battery swapping relies on the relation between operational costs and acquisition costs (Davis and Figliozzi, 2013). In this section, we compare the EMVRPTW-SMBS against solutions based on intra-route recharging by considering operational costs, vehicle costs and costs for siting CSs to document the business case for mobile battery swapping.

Operators of electric logistics fleets that tend to use solutions based on intra-route recharging currently decide on both station siting and vehicle routing (possibly simultaneously); therefore, it will not be sufficient to only focus on the minimisation of the total distance/driving time, and the minimisation of the number and cost of CSs sited and the total number of ECVs used must be also taken into account (Schiffer and Walther, 2017). This situation applies also to the case of mobile battery swapping in terms of the acquisition cost of ECVs and BSVs along with the total distance or travelling time incurred by them in the operational level. This paper is developed around the argument that there are multiple benefits in using BSVs in the design of delivery routes for ECVs which make this option an attractive line of research and practice:

- The cost for setting up a battery charging/swapping station can be significantly larger than the acquisition cost of a battery swapping van; Shao et al. (2017) compare the cost of a CS and a BSV across a same time-period, and claim that CSs can be over 10 times more expensive than battery swapping vans, when the cost of the required land and infrastructure, the cost of energy distribution and the cost of maintenance (employees and devices) are taken into account.

- $\quad$ A BSV can serve multiple ECVs in one BSV trip without any need to divert the route of the moving ECVs; consequently, a small number of BSVs may be required to serve multiple ECVs, and the total distance travelled by the ECVs can be expected to be significantly shorter than when ECVs must detour to visit CSs.

- The battery swapping service is carried out in a small fraction of the time required for recharging the ECV at a CS and hence the overall number and travel time of ECVs (and hence drivers' cost) is expected to be much smaller, compared to that from using CSs.

- Unlike CSs which once located cannot be moved, the use of BSVs provides a higher degree of flexibility for the logistic operator.

- ECVs must detour to visit CSs, but BSVs drive to ECVs. This may imply that a single BSV can potentially do the job of several CSs. Unlike a BSV, an opened CS may be accessible to only a few ECV routes, and it would be infeasible or too costly for other ECVs to divert their routes towards the opened CS for recharging. Therefore, comparatively more (potentially under-utilised) CSs must be opened in solutions based on intra-route recharging.

In support of these claims we have carried out several numerical experiments in section 5 of the paper; however, for illustration one instance of the problem is presented and discussed here. The chosen instance is instance C104-10 from the 'computational results' section of the paper, which is an instance with 10 customers and 4 potential CS sites (refer to section 5 for the details of the instance).

We solve the problem instance in two different settings; firstly, the problem is treated as an Electric Location Routing Problem with Time Windows and Partial Recharging (ELRPTW-PR) with a generalised cost function involving the opening cost of CSs, the acquisition cost of ECVs and the operational cost of the total distance travelled by the ECVs. In order to solve the problem in this setting, we have adapted the formulation proposed in Keskin and Çatay (2016) for the 
EVRPTW with partial recharging, and modified it to incorporate simultaneously the location aspects by assuming the available CS sites are potential sites to open. The resulting routes are shown in Figure 2.a, where one CS is opened and 4 ECVs are required to travel a total distance of 359.46. Secondly, the problem is treated as an EVRPTW-SMBS instance and is solved for the minimisation of a generalised cost function comprising the total acquisition cost of ECVs and BSVs and the cost of the total distance travelled by them using the mathematical formulation discussed in the next section. The resulting routes are presented in Figure 2.b. The solution yields one ECV and one BSV route in total, where the BSV meets the ECV at customer locations 7, 1, and 5, respectively, to swap the ECVs depleted battery with a fully charged one. As a result of this, the ECV travels a distance of 234.22 and the BSV travels a distance of 98.65; hence, a total distance of 332.87 by both the ECV and the BSV.
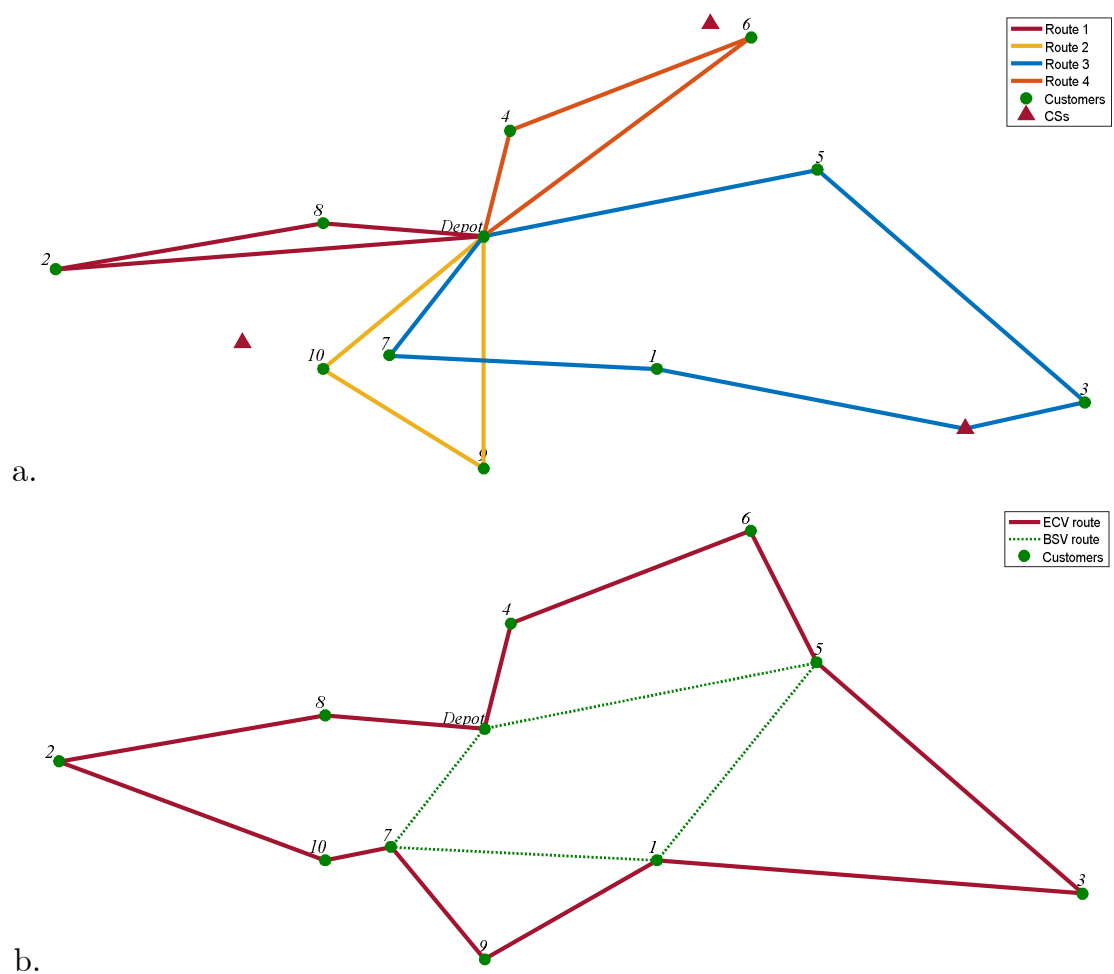

Figure 2 Optimal routes returned by (a) ELRPTW-PR and (b) EVRPTW-SMBS

Alongside these potential immediate benefits of a logistic design based on BSVs, in the longer run the flexibility of the possible solution and the agility brought about in the accomplishment of delivery tasks suggests that the EVRPTWSMBS is worth consideration.

It may be also worth discussing here an alternative technology to mobile battery swapping which corresponds to 'mobile fast recharging'. This recent technological option developed by companies such as NIO ${ }^{\circledR}$ and FREEWIRE TECHNOLOGIES $^{\circledR}$ (Figure 3) presents similar logistics opportunities as do the BSVs. For example, NIO ${ }^{\circledR}$ Power Mobile can serve as a mobile power bank that can travel anywhere to any EV in need of battery recharging and provide an extra $100 \mathrm{~km}$ with $10 \mathrm{~min}$ of charging ${ }^{2}$.
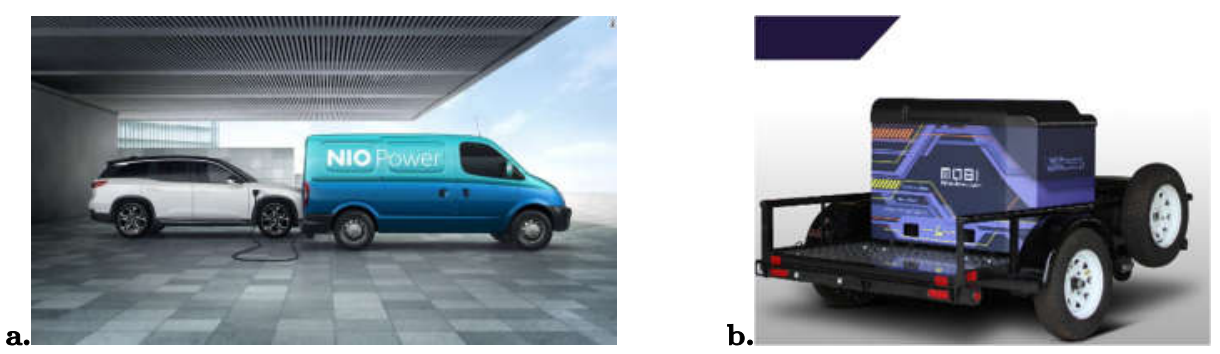

${ }^{2}$ https://www.nio.com/nio-power 
Figure 3 (a) NIO Power Mobile and (b) MOBI GEN TWS

While a logistic model similar to the one proposed in this paper for BSVs may be applicable for the case of Battery Recharging Vans (BRVs), we argue that BRVs require a dedicated investigation in future research due to several distinctive features. In particular, the difference in the servicing capacity and servicing technology of a BSV and a BRV make the two problems different in terms of their modelling and solution requirements. A BSV can carry a certain number of fully charged batteries to a certain number of ECVs, and each ECV restarts its route with a fully charged battery following a swapping service provided by a BSV. However, the servicing capacity of a BRV is itself dependent on how much recharge it provides to each ECV it visits, and each ECV may only need partial recharging to complete its delivery route. Also, the service time of a BSV is different from the service time of a BRV; a BSV requires a fixed amount of time to complete its service, but a BRV's service time is not known a priori as it depends on the amount of charging it provides. Therefore, the electric vehicle routing problem with mobile battery recharging is a different problem variant with different challenges which is worth exploring in future research.

Next, we propose a mathematical formulation for the EVRPTW-SMBS.

\subsection{Mathematical formulation of the problem}

The MILP formulation of the EVRPTW-SMBS works with the following decision variables:

- $x_{i j}$ : Binary variable equal to 1 iff arc $(i, j) \in A$ is traversed by an ECV.

- $z_{i j}$ : Binary variable equal to 1 iff $\operatorname{arc}(i, j) \in A$ is traversed by a BSV.

- $\delta_{i}$ : Binary variable equal to 0 iff battery swapping service at node $i \in N$ starts during $\left[y_{i}, e_{i}-s\right]$, and 1 otherwise (refer back to expression (1)).

- $y_{i}$ : Continuous variable denoting the time of arrival of an ECV at node $i \in N$.

- $a_{i}$ : Continuous variable denoting the service start time by an ECV at node $i \in N$.

- $w_{i}$ : Continuous variable denoting the time of arrival of a BSV at node $i \in N$.

- $\theta_{i}$ : Continuous variable denoting the battery swapping service start time by a BSV at node $i \in N$.

- $f_{i}$ : Continuous variable denoting the remaining load on an ECV upon arrival at node $i \in N$.

- $h_{i}$ : Integer variable denoting the number of the remaining fully-charged batteries on the BSV upon arrival at node $i \in N$.

- $u_{i}$ : Continuous variable denoting the remaining battery charge level of an ECV on arrival at node $i \in N$.

- $v_{i}$ : Continuous variable denoting the remaining battery charge level of a BSV on arrival at node $i \in N$.

The mathematical formulation of the EVRPTW-SMBS is given by (2)-(21):

$\min \mathcal{C}_{e} \sum_{j \in N_{\sigma}} x_{0 j}+\mathcal{C}_{b} \sum_{j \in N_{\sigma}} z_{0 j}+\mathcal{C}_{d_{e}} \sum_{(i, j) \in A} d_{i j} x_{i j}+\mathcal{C}_{d_{b}} \sum_{(i, j) \in A} d_{i j} z_{i j}$

Subject to:

$$
\begin{aligned}
& \sum_{j \in N_{\sigma}} x_{i j}=1, \quad \forall i \in C \\
& \sum_{j \in N_{0}} x_{j i}-\sum_{j \in N_{\sigma}} x_{i j}=0, \quad \forall i \in C \\
& \sum_{j \in N_{0}} z_{j i}-\sum_{j \in N_{\sigma}} z_{i j}=0, \quad \forall i \in C \\
& a_{i}=\max \left\{e_{i}, y_{i}\right\}, \quad \forall i \in C \\
& a_{i}+\left(t_{i j}+s_{i}\right) x_{i j}+\delta_{i} s-\left(l_{0}+s\right)\left(1-x_{i j}\right) \leq y_{j}, \quad \forall i \in N_{0}, j \in N_{\sigma}, i \neq j
\end{aligned}
$$




$$
\begin{aligned}
& e_{i} \leq a_{i} \leq l_{i}, \quad \forall i \in N \\
& a_{i}+s_{i}-\left(1-\delta_{i}\right)\left(l_{i}+s_{i}\right) \leq \theta_{i} \leq a_{i}+s_{i}+\left(1-\delta_{i}\right)\left(l_{i}+s_{i}\right), \quad \forall i \in C \\
& y_{i} \leq \theta_{i} \leq\left(1-\delta_{i}\right)\left(e_{i}-s\right)+\left(1-\sum_{j \in N_{\sigma}} z_{i j}+\delta_{i}\right)\left(l_{i}+s_{i}\right), \quad \forall i \in C \\
& w_{i} \leq \theta_{i}, \quad \forall i \in C \\
& \theta_{i}+\left(t_{i j}+s\right) z_{i j}-l_{0}\left(1-z_{i j}\right) \leq w_{j}, \quad \forall i \in N_{0}, j \in N_{\sigma}, i \neq j \\
& 0 \leq f_{j} \leq f_{i}-\left(q_{i} x_{i j}\right)+Q_{e}\left(1-x_{i j}\right), \quad \forall i \in N_{0}, j \in N_{\sigma}, i \neq j \\
& 0 \leq f_{0} \leq Q_{e} \\
& 0 \leq h_{j} \leq h_{i}-z_{i j}+Q_{b}\left(1-z_{i j}\right), \quad \forall i \in N_{0}, j \in N_{\sigma}, i \neq j \\
& 0 \leq h_{0} \leq Q_{b} \\
& 0 \leq u_{j} \leq u_{i}-\left(r_{e} d_{i j} x_{i j}\right)+\left(B_{e} \sum_{\rho \in N_{0}: j \in C} z_{\rho j}\right)+B_{e}\left(1-x_{i j}\right), \quad \forall i \in C, j \in N_{\sigma}, i \neq j \\
& 0 \leq u_{i} \leq B_{e}, \quad \forall i \in N \\
& u_{i} \geq \sum_{j \in N_{5}} r_{e} d_{i j} x_{i j}, \quad \forall i \in C \\
& 0 \leq v_{j} \leq v_{i}-\left(r_{b} d_{i j} z_{i j}\right)+B_{b}\left(1-z_{i j}\right), \quad \forall i \in N_{0}, j \in N_{\sigma}, i \neq j \\
& 0 \leq v_{i} \leq B_{b}, \quad \forall i \in N
\end{aligned}
$$

Expression (2) is the objective function that seeks to minimise the total cost consisting of the acquisition cost of ECVs and BSVs and the operational cost of the total distance travelled by them. Constraints (3) to (5) are routing constraints, constraints (6) to (12) are scheduling and synchronisation constraints, constraints (13) to (16) are capacity constraints, and finally, constraints (17) to (21) are battery level control and swapping determination constraints. These constraints are further detailed below.

Constraints (3) indicate that each customer must be visited exactly once by an ECV for delivery. Constraints (4) and (5) together guarantee that if a vehicle (i.e. an ECV or a BSV) enters a customer node, it also exits.

Constraints (6) denote the service start time at each customer. Constraints (7) determine the arrival time of an ECV at each node by accounting for the service start time at the upstream node, its service time, and possibly its required time for a requested swap by a BSV. Constraints (8) ensure that customers' service starts during their time windows. Constraints (9) and (10) determine if the battery swapping service begins before or after the customer service (refer back to expression (1)). Constraints (11) present the possible arrival time of the BSV at the designated point of swapping; i.e. on or before the decided swapping service start time. Constraints (12) determine the arrival time of a BSV at each node by accounting for the swapping service start time at the upstream node plus the service time. 
Constraints (13) and (14) ensure demand fulfilment while guaranteeing that the capacity of the ECVs is not violated, and constraints (15) and (16) do the same for BSVs. Constraints (17) to (19) determine the battery charge level of ECVs after visiting a customer and in case a battery swapping service is carried out. Note that both constraints (17) and (18) are needed to accurately determine the battery level of an ECV in case a battery swapping by a BSV is scheduled. The use of constraints (17) alone is not sufficient, as in the case of non-zero $u_{i}$, by battery swapping the battery capacity will be violated without (18). Finally, constraints (20) and (21) ensure that the battery charge of a BSV never falls below 0.

We use this formulation to solve exactly small sized instances of the EVRPTW-SMBS with up to 25 customer nodes. The cost of these solutions is compared with the cost of solutions based on intra-route recharging. We also use the solution to these instances as a benchmark for the evaluation of the performance of the proposed solution algorithm presented in the next section.

\section{The algorithm}

The EVRPTW-SMBS is very difficult to solve to optimality in case of realistically sized problem instances and hence the development of tailored heuristic solution algorithms to tackle practical problem sizes is important. Solving the EVRPTWSMBS heuristically, however, is also a significant challenge due to the spatio-temporal synchronisation requirement and the existence of the compound interdependence problem discussed earlier. In the EVRPTW-SMBS, routing must be carried out in two levels for the ECVs and BSVs such that BSVs are present at a designated time and location to swap the depleted battery on an ECV with a fully charged one. Any slight change in the routes of one level can change the routes of the other level and therefore complicate solution evaluation in the course of the local search.

In this section, we propose a methodology for exact evaluation of each given solution in the context of the EVRPTWSMBS. This is a two-stage routine based on the integration of a Dynamic Programming (DP) algorithm in the first stage with an Integer Program (IP) for label selection in the second stage, referred to as the "DPIP". Placing the DPIP 'wisely' at the heart of the local search as the routine for solution evaluation, it is possible to tackle EVRPTW-SMBS efficiently. We develop and use an ILNS algorithm as the higher-level heuristic and put forth three versions of the algorithm that use different solution evaluation routines at their core. In the following, a high-level exposition of the proposed ILNS is first provided, and then the DPIP and the three extensions of the DP-ILNS are described.

Prior to discussing the proposed algorithms, however, we need to establish that in the rest of this section whenever we refer to a 'Solution', it is meant to be a set $\mathcal{S}=\left\{\mathcal{R}_{1}, \mathcal{R}_{2}, \ldots, \mathcal{R}_{k}\right\}$, which is composed of $k$ capacity feasible ECV routes $\mathcal{R}_{b}, \forall b \in\{1, \ldots, k\}$ visting all customers in $C$ exactly once. Each route $\mathcal{R}_{b}=\left\{c_{0}, c_{1}, \ldots, c_{\ell}, c_{\sigma}\right\}$, on the other hand, is a sequence of customer visits for a given ECV that starts at the depot $\left\{c_{0}\right\}$, visits a set of customers $\left\{c_{1}, \ldots, c_{\ell}\right\}$ and terminates at the final depot $\left\{c_{\sigma}\right\}$. Therefore, a solution only contains information about a set of ECV routes denoting the sequence of customer visits and does not imply any other information regarding the need for the battery swapping services. Correspondingly, by '(Optimal) Evaluation of a Solution', we mean to determine (optimally) the schedules for the required battery swapping services.

\subsection{The higher-level heuristic}

To tackle the EVRPTW-SMBS, we develop and use an ILNS algorithm as the higher-level heuristic. The LNS (Shaw, 1998) is a conceptually simple metaheuristic which has proven successful in solving different variants of routing problems, particularly the VRPTW. It is based on large rearrangements in a current solution by applying several removal and reinsertion heuristics, and hence moving from one area within the feasible region to another using rather large steps. For brevity, we avoid elaborating on the details of the LNS and its well-known adaptive extension, i.e. ALNS (Ropke and Pisinger, 2006) here and refer the reader to the original studies of Shaw (1998) and Ropke and Pisinger (2006) for that purpose.

In order to achieve a better exploitation capability, the LNS can be further equipped with an auxiliary intensification procedure. In this study, we propose to use a Simulated Annealing (SA) metaheuristic with a new neighbourhood exploration strategy as the solution intensifier, which is only invoked upon finding local optima to seek the possibility of finding a better solution. An overview of the proposed ILNS algorithm is given in Algorithm 1.

In the first step of the proposed algorithm (line 1) a solution $\mathcal{S}_{0}$ is generated using a simple heuristic. This heuristic puts all customers into a "non-routed" set and initiates an empty ECV route in the beginning. Then, in each iteration the algorithm extracts a customer from the non-routed set and assuming an unlimited battery capacity tries to insert it at its best location 
in the current route(s) with respect to the total ECV distance travelled. If the customer cannot be put into any of the existing routes due to vehicle capacity or time windows constraints, a new route is initiated to accommodate the customer. This procedure is iterated until the non-routed set is empty.

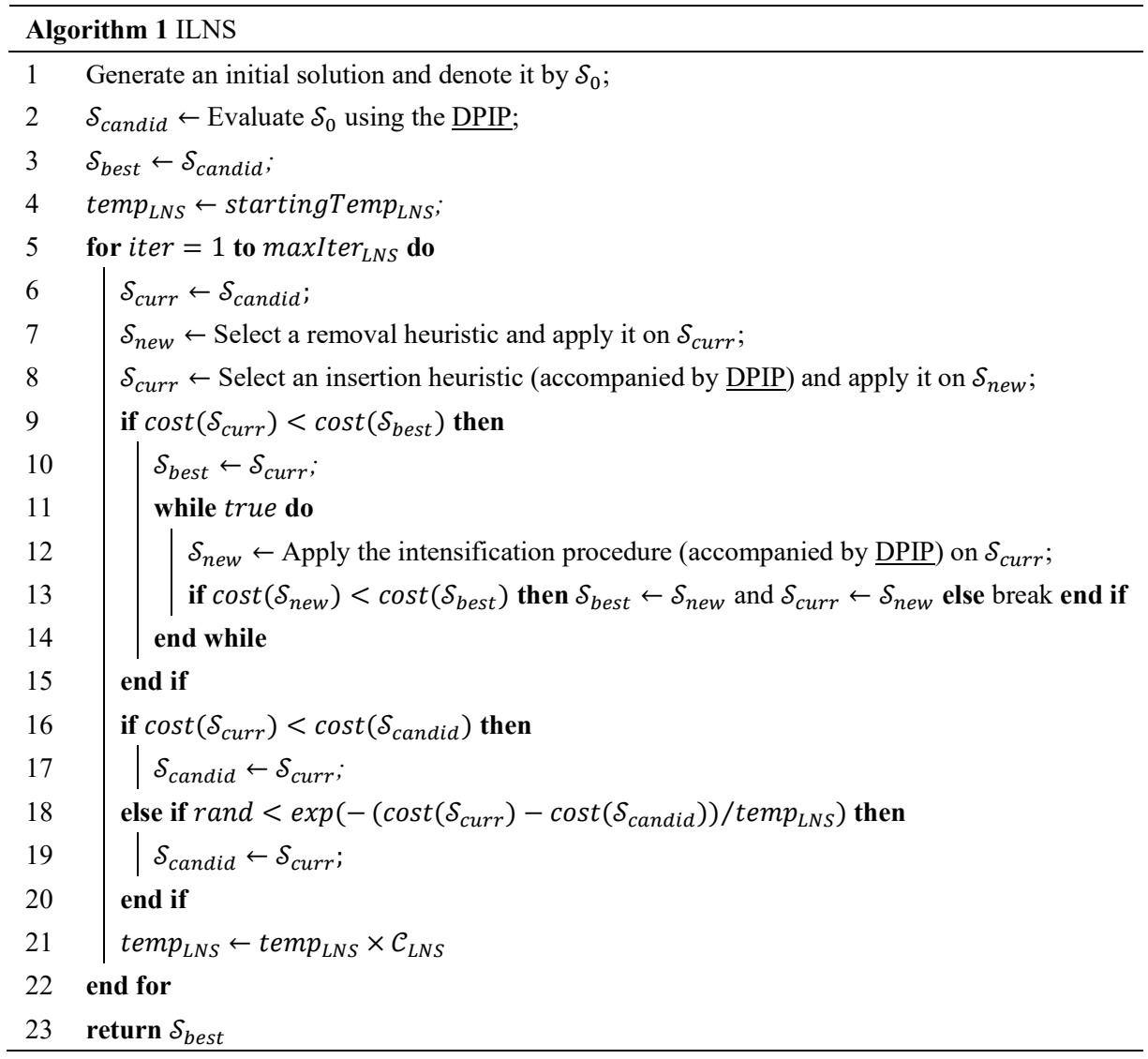

Following the generation of the initial solution $\mathcal{S}_{0}$, it is optimally evaluated using the DPIP (discussed in the next subsection) and the optimal evaluation is called $\mathcal{S}_{\text {candid }}$ (line 2). Henceforth, the proposed ILNS takes a fixed number of iterations $\left(\right.$ maxIter $\left._{L N S}\right)$ to seek a better solution $\delta_{\text {best }}$. In each iteration, a removal heuristic is selected from a set of available removal heuristics and is applied on the current solution $\mathcal{S}_{\text {curr }}$ to remove a certain number of customers from the routes in the solution (line 7). We are using three removal heuristics: (i) Shaw removal (Shaw, 1998), (ii) random removal (Ropke \& Pisinger, 2006), and (iii) worst removal (Ropke \& Pisinger, 2006), all with equal probability to be selected. For all these removal heuristics, the number of customers to remove is determined by selecting a random integer in the interval $\left[4, n \rho_{\text {rem }}\right]$, where $\rho_{\text {rem }} \in[0,1]$ is a user defined parameter. In addition to this parameter, there is a $\rho_{\text {shaw }} \in \mathbb{R}_{+}$parameter for the Shaw removal which controls determinism in the relatedness function (see Shaw, 1998), and there is a $\rho_{\text {worst }} \in \mathbb{R}_{+}$ parameter for the worst removal that controls the degree of randomisation (see Ropke and Pisinger, 2006).

The destroyed solution $\mathcal{S}_{\text {new }}$ after applying the selected removal heuristic, and the set containing removed customers are then submitted to a selected re-insertion algorithm to repair $\mathcal{S}_{\text {new }}$ and retrieve a possibly new $\mathcal{S}_{\text {curr }}$ (line 8 ). We use two insertion heuristics adopted from Ropke and Pisinger (2006) for this purpose: (i) regret-2 heuristic, and (ii) regret-2 heuristic with noise (see Ropke and Pisinger, 2006 for details). There is only one parameter here (i.e. $\eta$ ) associated with the second heuristic to control the amount of noise.

It is very important to note that unlike other VRPs, in the context of the EVRPTW-SMBS routes are not independent from one another and insertion of a customer in a route affects the entire solution (i.e. the set of ECV and BSV routes) and its evaluation. Therefore, a key in the design of the algorithm is to decide how the value of re-insertions here in the repair step of the proposed ILNS and in the intensification step (line 12) is determined. For now, suppose that each time an insertion occurs the new solution is submitted to DPIP for optimal evaluation. It is clear that this is a computationally 
intensive and prohibitive requirement, but we later discuss how in the extensions of the algorithm this is relaxed using lexicographic decomposition techniques.

Following the application of the destroy and repair mechanisms in lines 7 and 8 of the algorithm, the intensification procedure is invoked if the new resulting solution $\mathcal{S}_{\text {curr }}$ is lower cost than the existing best solution $\mathcal{S}_{\text {best }}$ (line 8). After updating $\mathcal{S}_{\text {best }}$ (line 10), as long as $\mathcal{S}_{\text {best }}$ can be improved, the intensification procedure is repeatedly applied on $\mathcal{S}_{\text {best }}$. The structure of the intensification procedure is similar to the successful SA algorithm proposed by Bent and Van Hentenryck (2004) for VRPTW, and mainly differs in its neighbourhood exploration strategy, which is indeed the special feature of their SA (and hence ours). In each iteration of their SA algorithm, Bent and Van Hentenryck (2004) choose randomly a move operator and a customer, and then consider all the possible moves for this customer using the selected operator to see if any improvement could be found. While we use the same 5 well- known local search operators that Bent and Van Hentenryck (2004) use in their study; i.e. 2-opt, Or-opt, Relocation, Swap, and Crossover, we explore a wider subneighbourhood by selecting $\lambda n$ customers instead of only one customer, where the rate $\lambda \in[0,1]$ is a user defined parameter. The closer is the selected $\lambda$ to 1 , the wider will be the explored sub-neighbourhood, and thus the better might be the ultimate solution, but also the slower would be the overall algorithm. Note that the SA algorithm used in the intensification procedure requires 5 other input parameters corresponding to timeLimit INT, $\mathcal{C}_{\text {INT }}$, tempLimit INT $_{\text {, }}$ startingTemp $_{I N T}$, and maxIter $_{I N T}$ (see Appendix A. and Bent and Van Hentenryck, 2004 for details).

In lines 16 to 20 of the algorithm, $\mathcal{S}_{\text {curr }}$ is compared with $\mathcal{S}_{\text {candid }}$, and if $\mathcal{S}_{\text {curr }}$ is not an improvement over $\mathcal{S}_{\text {candid }}$, an SA-wise acceptance criterion is used to examine if $\mathcal{S}_{\text {candid }}$ could be updated. Note that the temperature temp $p_{L N S}$ in the algorithm starts out at startingTemp $p_{L N S}$ (line 4) and is decreased at the end of every iteration using the expression temp $p_{L N S} \leftarrow$ temp $p_{L N S} \times \mathcal{C}_{L N S}$ (line 21 ), where $0<\mathcal{C}_{L N S}<1$ is the cooling rate.

We next describe the DPIP procedure used for the exact evaluation of a given solution in the context of the EVRPTWSMBS.

\subsection{Optimal evaluation of an ECV route in EVRPTW-SMBS}

As was defined earlier, optimal evaluation of a given solution $\mathcal{S}$ (a set of capacity feasible ECV routes each representing the sequence of customer visits) in the context of the EVRPTW-SMBS corresponds to the optimal determination of the required battery swapping services such that $\mathcal{S}$ is turned into a feasible EVRPTW-SMBS solution and objective function (2) is minimised. Due to the interdependence between ECV and BSV routes, this is a complicated task and, unlike in typical VRPs, following any change in a given route $\mathcal{R}_{b} \in \mathcal{S}$ in the course of the local search, the entire $\mathcal{S}$ must be re-evaluated, and not just $\mathcal{R}_{b}$.

We propose an exact two-stage evaluation function to carry out this task: in the first stage we use a DP to generate a set of non-dominated labels at the endpoint of each energy infeasible route; these non-dominated labels correspond to 'incomparable' ways for requesting battery swapping at different locations and times along the route, using any of which the corresponding energy infeasible route can be turned into an energy feasible one. The generated labels for all routes are then submitted to an IP to combine and generate an optimal EVRPTW-SMBS evaluation of the given solution. These two stages are detailed in the following.

The proposed DP for the first stage is presented in Algorithm 2. This algorithm takes the graph $G$, an ECV route $\mathcal{R}=$ $\left\{c_{0}, c_{1}, \ldots, c_{\sigma}\right\}$ (where $c_{0}$ and $c_{\sigma}$ are the depot), and the time-windows and service times of the customers on the route in its input (line 1), and returns a set of non-dominated labels $\mathcal{L}_{\sigma}$ at the destination node of the given route (i.e. at the final depot). Along with $\mathcal{L}_{\sigma}$, and associated with each label in $\mathcal{L}_{\sigma}$, the algorithm returns also information about the customers that require a swapping service in $\mathcal{G}_{\sigma}$, and the time (or time interval) at/during which these customers need the service to be available in $\mathcal{F}_{\sigma}$ (line 27). This information must be passed to the second stage IP solver of the algorithm for combination of labels.

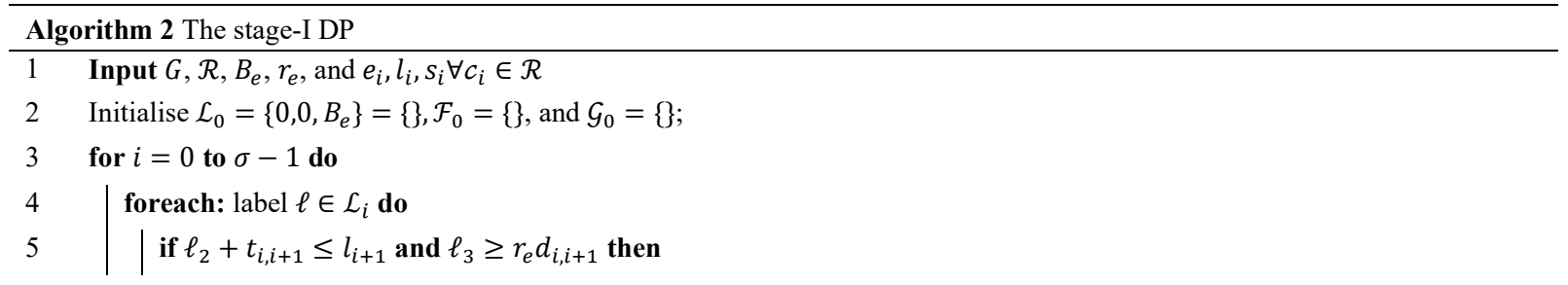




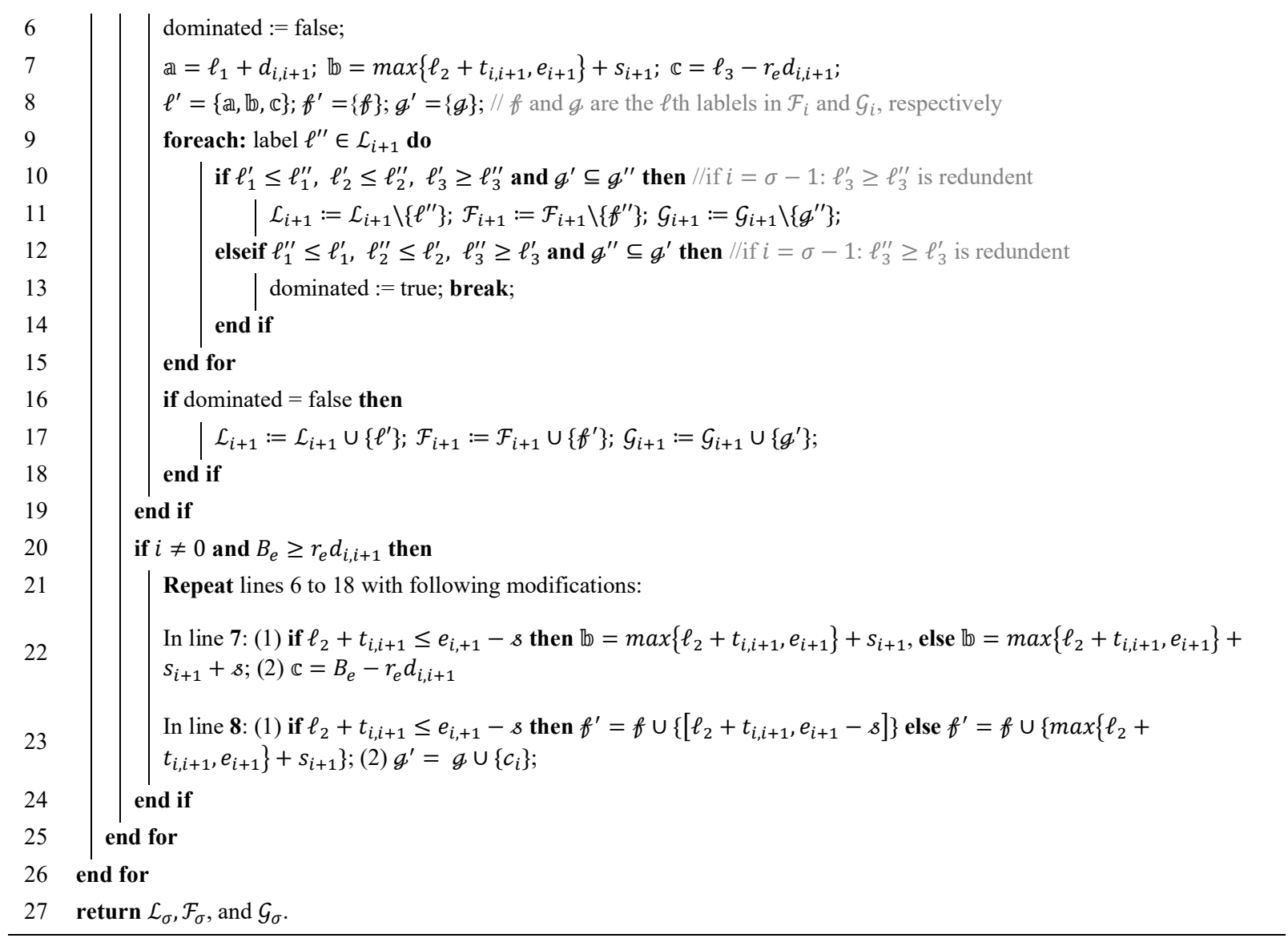

The algorithm retains and extends a set of labels $\mathcal{L}_{i}, \mathcal{F}_{i}$, and $\mathcal{G}_{i}$ at each node $c_{i}$ along $\mathcal{R}$. Each label $\ell \in \mathcal{L}_{i}$ is a tuple of length 3 , where $\ell_{1}$ stores the accumulated distance, $\ell_{2}$ stores the accumulated travel time (this includes the travel time of the ECV, the potential waiting time for time window's opening and the accumulated overhead time for swapping services), and $\ell_{3}$ stores the available battery charge level up to the current node in $\mathcal{R}$. Each monitoring label $g \in \mathcal{G}_{i}$ and f $\in \mathcal{F}_{i}$, on the other hand, is an open-ended list of customers requiring swaps and their requested service time/time intervals, respectively. The first set of labels at $c_{0}$ is initiated in line 2 of the algorithm and it is extended in lines 3 to 26 of the algorithm. If no battery swapping is taking place at node $c_{i+1}$, the extension of the travel time from node $c_{i}$ to $c_{i+1}$ via label $\ell$ follows the expression below (line 7 of the algorithm):

$$
\ell_{2}^{\prime}=\max \left\{\ell_{2}+t_{i, i+1}, e_{i+1}\right\}+s_{i+1}
$$

where $\ell_{2}^{\prime}$ is the travel time (ready time) at node $c_{i+1}$ and $\ell_{2}$ is the ready time at node $c_{i}$.

However, if battery swapping is taking place at node $c_{i+1}$, two situations are possible: if the arrival of the ECV is 'well' before the customer's time window, i.e. $\ell_{2}+t_{i, i+1} \leq e_{i+1}-s$, then the same expression (22) is used; otherwise, the extension follows expression (23) below (line 22 of the algorithm):

$$
\ell_{2}^{\prime}=\max \left\{\ell_{2}+t_{i, i+1}, e_{i+1}\right\}+s_{i+1}+s
$$

In the former case, the algorithm passes the time interval $\left[\ell_{2}+t_{i, i+1}, e_{i+1}-s\right]$ as a potential time window for the BSV arrival time that must be decided at the next stage of the algorithm, and in the latter case no time interval flexibility is granted and the BSV must be available for the battery swapping service at time $\max \left\{\ell_{2}+t_{i, i+1}, e_{i+1}\right\}+s_{i+1}($ line 23).

Clearly, the distinctive feature of the proposed DP that particularly leads to extra information regarding the need for swapping services corresponds to lines 20 to 25 of the algorithm, where the restriction on the available battery charge level is lifted and it is assumed that the ECV is ready to depart the node using a fully charged battery as a result of a potential battery swapping service by a BSV. Comparison of two labels in the context of this algorithm and the development of 
efficient domination rules, however, is not straightforward. A label with shorter distance and travel time and larger battery charge level does not necessarily dominate another label with a larger travel time and smaller battery charge level. This is because the total distance travelled by the BSVs also contributes to the objective function and the length of the routes travelled by BSVs is determined by the customers they must visit. Therefore, the associated battery swapping requesting customers to each label make them incomparable. It is not even possible to say a label with fewer battery swapping requesting customers is better, because it might be a shorter route for the BSV to visit 3 customers which are close to each other and to the depot, rather than a single customer too far from the depot, for example.

We handle this situation by engaging labels $g \in \mathcal{G}_{i}$ in the development of the domination rule (lines 10 and 12 ):

Remark 1 If for two labels $\ell^{\prime}$ and $\ell^{\prime \prime}$ in $\mathcal{L}_{i}$, and their corresponding monitoring labels $g^{\prime}$ and $g^{\prime \prime}$ in $\mathcal{G}_{i}$, we have $\ell_{1}^{\prime} \leq$ $\ell_{1}^{\prime \prime}, \ell_{2}^{\prime} \leq \ell_{2}^{\prime \prime}, \ell_{3}^{\prime} \geq \ell_{3}^{\prime \prime}$, and $g^{\prime} \subseteq g^{\prime \prime}$, then $\ell^{\prime} \leqslant \ell^{\prime \prime}$ (i.e. label $\ell^{\prime}$ dominates label $\ell^{\prime \prime}$ ).

Based on remark 1, if for example we have three partial paths $\mathrm{A}, \mathrm{B}$, and $\mathrm{C}$, with exactly the same distance, travel time and battery charge level, and with associated swapping location sets of $\{1,2\}$, $\{1\}$, and $\{2,3\}$, respectively, B dominates $\mathrm{A}$, but $\mathrm{B}$ and $\mathrm{C}$, and $\mathrm{A}$ and $\mathrm{C}$ are incomparable. The clear explanation for this is the triangular inequality which implies that visiting an additional customer in the second level by a BSV will only lead to extra distance travelled.

In order to illustrate better the working of the proposed DP in an EVRPTW-SMBS instance, we use here instance C101-5 from section 5 of the paper as an example. In the context of this instance, suppose that in the course of the local search we intend to evaluate optimally a generated solution $\mathcal{S}=\{[0,1,2,0],[0,4,3,0],[0,5,0]\}$ which is a solution with three capacity-feasible ECV routes. Due to the given battery capacity of an ECV in this instance, routes 1 and 2 (i.e. [0,1,2,0] and $[0,4,3,0])$ are energy infeasible and the ECVs operating them need the battery swapping service over their route. The application of the proposed DP in evaluating route $[0,1,2,0]$ is illustrated in Figure 4 . The figure illustrates the extension of the labels along the given ECV route, and shows that at the final node, we must choose among 2 non-dominated labels only. The path corresponding to the first label involves a battery swapping at customer 2 which can take place any time in the interval [569.41,649], and the path corresponding to the second label involves a swapping at customer 1 which can take place any time in the interval $[51.03,446]$. The third label is clearly a dominated label based on Remark 1.

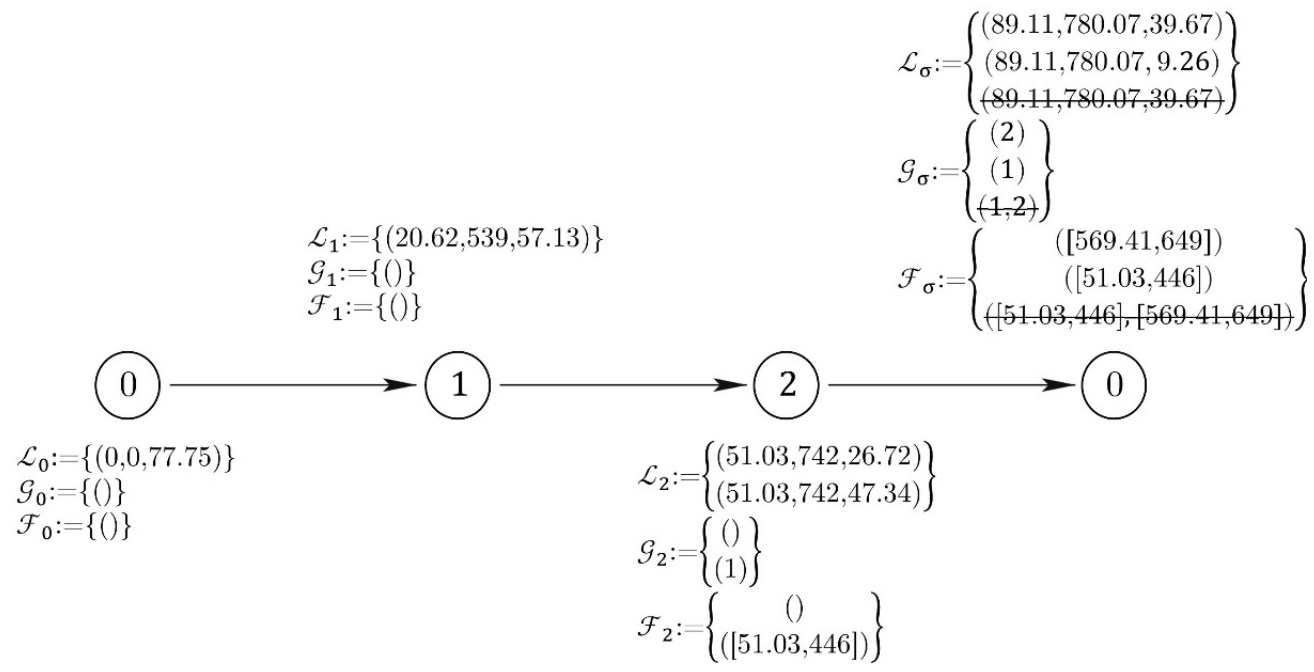

Figure 4 An illustrative example for the proposed DP

In order to illustrate later how the second stage IP combines the resulting labels, Figure 5 shows also the resulting $\mathcal{L}_{\sigma} \mathrm{s}$ after the application of the first stage DP on routes $[0,4,3,0]$ and $[0,5,0]$ in $\mathcal{S}$. 


$$
\begin{array}{ll}
\text { Route }[0-4-3-0] & \text { Route }[0-5-0] \\
\mathcal{L}_{\sigma}:=\left\{\begin{array}{l}
(95.99,804.26,39.67) \\
(95.99,804.26,11.49)
\end{array}\right\} & \begin{array}{l}
\mathcal{L}_{\sigma}:=\{(43.08,743.54,34.67)\} \\
\mathcal{G}_{\sigma}:=\{()\}
\end{array} \\
\mathcal{G}_{\sigma}:=\left\{\begin{array}{l}
(3) \\
(4)
\end{array}\right\} & \begin{array}{l}
\mathcal{F}_{\sigma}:=\{()\} \\
\mathcal{F}_{\sigma}:=\left\{\begin{array}{c}
(29.73,552]) \\
(763.18)
\end{array}\right\}
\end{array}
\end{array}
$$

Figure 5 The EPIP-based DP endpoint labels of the routes in the example

Having identified the final set of non-dominated endpoint labels at each route and their corresponding attributes, the algorithm can proceed to the second stage and select an optimal combination of these labels using a simple integer linear programming model that is described in the following. Hereafter, we refer to this problem as the Label Selection Problem (LSP).

To develop the LSP, the following sets and parameters must be defined following the outputs from the Stage-I DP: Sets:

- $\mathcal{N}=\left\{c_{1}, c_{2}, \ldots, c_{n}\right\}:$ The unique set of customers requiring battery swaps in all the identified $\mathcal{G}_{\sigma} \mathrm{s}$ of all routes (in the example, we have $\mathcal{N}=\{1,2,3,4\}$ ).

- $\mathcal{S}=\left\{\mathcal{R}_{1}, \mathcal{R}_{2}, \ldots, \mathcal{R}_{k}\right\}$ : The index set of all routes in the solution (in the example, we have $\mathcal{S}=\{1,2,3\}$, where index 1 refers to route 1 , i.e. route $[0,1,2,0]$, index 2 refers to route 2 , i.e. route $[0,4,3,0]$, and index 3 refers to route 3 , i.e. route $[0,5,0])$.

- $\mathcal{L}=\left\{\ell_{1}, \ell_{2} \ldots, \ell_{m}\right\}$ : the ordered set containing indices pointing to all identified non-dominated labels $\mathcal{L}_{\sigma} \mathrm{s}$ (in the example, we have $\mathcal{L}=\{1,2,3,4,5\}$, where index 1 and index 2 refer to labels at the endpoint of route 1, indices 3 and 4 refer to labels at the endpoint of route 2, and index 5 refers to the label at the endpoint of route 3 ).

- $\quad$ For ease of reference, assume that $\mathcal{N}_{0}=\{0\} \cup \mathcal{N}, \mathcal{N}_{\sigma}=\mathcal{N} \cup\{\sigma\}$, and $\mathcal{A}=\left\{(i, j) \mid i \in \mathcal{N}_{0}, j \in \mathcal{N}_{\sigma}, i \neq j\right\}$. Parameters:

- $\quad V_{\ell r}:$ A parameter equal to 1 if label $\ell \in \mathcal{L}$ belongs to route $r \in \mathcal{S}$, and 0 otherwise.

- $\mathcal{U}_{\ell i}$ : A parameter equal to 1 if label $\ell \in \mathcal{L}$ contains customer $i \in \mathcal{N}$, and 0 otherwise.

- $\underline{\mathcal{T}}_{i}$ : The earliest time a BSV can start service at customer $i \in \mathcal{N}$ on label $\ell \in \mathcal{L}$. Note that if $\mathcal{U}_{\ell i}=0$, then $\underline{\mathcal{T}_{p i}}=0$.

- $\quad \overline{\mathcal{T}}_{\ell i}$ : The latest time a BSV can start service at customer $i \in \mathcal{N}$ on label $\ell \in \mathcal{L}$. Note that if $\mathcal{U}_{\ell i}=0$, then $\overline{\mathcal{T}}_{\ell i}=0$. Also note that if swapping must take place after ECV serves the customer (i.e. a time instant and not a range in the corresponding $\mathcal{F}$ label), then $\underline{\mathcal{T}}_{\ell i}=\overline{\mathcal{T}}_{\ell i}$.

- $\mathcal{D}_{\ell}$ : the total distance associated with label $\ell \in \mathcal{L}$.

For illustration, the defined parameters in the case of the example are given below:

$$
\mathcal{V}=\left[\begin{array}{lll}
1 & 0 & 0 \\
1 & 0 & 0 \\
0 & 1 & 0 \\
0 & 1 & 0 \\
0 & 0 & 1
\end{array}\right], \quad \mathcal{\quad}=\left[\begin{array}{llll}
0 & 1 & 0 & 0 \\
1 & 0 & 0 & 0 \\
0 & 0 & 1 & 0 \\
0 & 0 & 0 & 1 \\
0 & 0 & 0 & 0
\end{array}\right], \quad \mathcal{T}=\left[\begin{array}{cccc}
0 & 569.41 & 0 & 0 \\
51.03 & 0 & 0 & 0 \\
0 & 0 & 29.73 & 0 \\
0 & 0 & 0 & 763.18 \\
0 & 0 & 0 & 0
\end{array}\right], \quad \overline{\mathcal{T}}=\left[\begin{array}{cccc}
0 & 649 & 0 & 0 \\
446 & 0 & 0 & 0 \\
0 & 0 & 552 & 0 \\
0 & 0 & 0 & 763.18 \\
0 & 0 & 0 & 0
\end{array}\right]
$$

$\mathcal{D}=\left[\begin{array}{lllll}89.11 & 89.11 & 95.99 & 95.99 & 43.08\end{array}\right]$

Finally, the LSP uses the following decision variable, alongside $z_{i j}, \theta_{i}, h_{i}$ and $v_{i}$ defined earlier in the paper:

- $\quad x_{\ell}$ : Binary variable equal to 1 iff label $\ell \in \mathcal{L}$ is selected.

The LSP is given below:

$\operatorname{Min} \mathcal{C}_{b} \sum_{i \in \mathcal{N}_{\sigma}} z_{0 i}+\mathcal{C}_{d_{b}} \sum_{(i, j) \in A} d_{i j} z_{i j}+\mathcal{C}_{d_{e}} \sum_{\ell \in \mathcal{L}} \mathcal{D}_{\ell} x_{\ell}$

Subject to: 


$$
\begin{aligned}
& \sum_{\ell \in \mathcal{L}} \mathcal{V}_{\ell r} x_{\ell}=1, \quad \forall r \in \mathcal{S} \\
& \sum_{\ell \in \mathcal{L}} u_{\ell i} x_{\ell} \leq 1, \quad \forall i \in \mathcal{N} \\
& \sum_{j \in \mathcal{N}_{1}} z_{i j}=\sum_{\ell \in \mathcal{L}} u_{\ell i} x_{\ell}, \quad \forall i \in \mathcal{N} \\
& \sum_{\ell \in \mathcal{L}} \underline{\mathcal{T}}_{\ell i} x_{\ell} \leq \theta_{i} \leq \sum_{\ell \in \mathcal{L}} \overline{\mathcal{T}}_{\ell i} x_{\ell}, \quad \forall i \in \mathcal{N}
\end{aligned}
$$

and (5), (15), (16), (20) and (21) (in these constraints replace $C, N_{0}$ and $N_{\sigma}$, with $\mathcal{N}, \mathcal{N}_{0}$ and $\mathcal{N}_{\sigma}$, respectively).

In the proposed LSP, the objective function (24) minimises the acquisition cost of BSVs (first term) and the cost of the total distance travelled by the BSVs and the ECVs (second and third terms). Constraints (25) ensure that exactly one label is selected from the endpoint labels of each route. Constraints (26) guarantee each customer exists no more than once on the selected labels. Constraints (27) ensure that a BSV will be visiting a selected customer location. Finally, constraints (28) determine the BSVs service start time at the selected customer locations.

The LSP is a simple IP to solve explicitly, but calling the full DPIP over every newly generated solution in the course of the algorithm is computationally expensive. Therefore, in the next subsection, we propose three different algorithms that decompose the two stages of the DPIP in the course of the algorithm and only call the complete DPIP after finding certain local optima. It is also worth mentioning that one can derive a heuristic extension of the proposed DPIP, mainly by: (i) restricting the allowed size of the label sets at each node along the ECV route in the first stage DP (this is particularly useful in the case of the instances with wide time windows where routes visit a quite large number of customers), and (ii) by terminating the solver dealing with LSP prematurely (e.g. restricting the CPU time devoted to solving the LSP) or solving the LSP heuristically.

\subsection{Alternative methods for solution evaluation}

Due to the interdependence between ECV routes, and between the routes of the two levels, i.e. ECVs and BSVs, the global impact of a local change in the structure of an individual route cannot be determined by considering that route only. At the same time, evaluation of each EVRPTW-SMBS solution to optimality following the application of a move operator in the course of the local search using the proposed DPIP is quite costly. Hence, the DPIP must be reasonably used at certain points in the course of the solution algorithm only and the general solution evaluation should be assigned to a faster evaluation procedure.

To handle local alterations in routes in the local level, we propose to lexicographically decompose the two stages of the DPIP and incorporate an intuitive "look-ahead" element in the evaluation procedure. To do so, in the first stage, instead of returning the full set of non-dominated labels at the endpoint of each route and then submitting all these labels to the LSP solver, we apply stronger dominance rules and select only one label based on some criteria at the endpoint of each route, and hence eliminate the need for solving the LSP. We examine two different methods:

1) In the first method, we use "ECVs total distance travelled" as the intuitive look-ahead criterion; that is, we hypothesize that minimising the total distance of ECV routes might ultimately lead to a near optimal evaluation of the EVRPTW-SMBS. To do so, following each move and hence alteration in one of the existing ECV routes, we use a modified extension of the proposed DP, referred to as the DistBased-DP, for the affected route, and at the end-point select the label with minimum distance travelled. If two labels share exactly the same distance at the endpoint labels of the DistBased-DP, the label with fewer number of battery swapping services is selected. If this is also the same, one label is selected randomly.

2) In the second method, we use "total number of battery swapping services required" as the intuitive look-ahead criterion; that is, we hypothesize that minimising the total number of battery swapping services required by an ECV route might lead to fewer BSVs required and smaller total distance travelled by them, and hence a near optimal evaluation of the EVRPTW-SMBS. To do so, in the proposed DP (Algorithm 2), each label $\ell \in \mathcal{L}_{i}$ is extended to a tuple of length 4 , where $\ell_{4}$ stores the total number of battery swapping services required up to the current node in $\mathcal{R}$, and the domination rules in lines 10 and 12 of the algorithm are extended to include $\ell_{4}^{\prime} \leq \ell_{4}^{\prime \prime}$ 
and $\ell_{4}^{\prime \prime} \leq \ell_{4}^{\prime}$, respectively (we refer to this new DP as the BattBased-DP). Then, following each move and hence alteration in one of the existing ECV routes, we just use the BattBased-DP for the affected route and at the endpoint select the label with minimum number of battery swapping services. If two labels share exactly the same number of battery swapping services, the label with a smaller distance is selected. If this is also the same, one label is selected randomly.

Note that using these evaluation methods, with each move in the course of the algorithm only one (or at most two) routes must be evaluated and to determine the global impact of the move, unlike the case of the DPIP, there is no need to evaluate all other routes also. Therefore, one can use only these methods for solution evaluation and hence drive the search in the proposed DP-ILNS, and postpone the optimal evaluation using the DPIP until the final best solution is returned. For a much better performance, however, it is possible to incorporate reasonably several checkpoints into the course of the algorithm, where the DPIP intervenes and evaluates a current best solution to optimality. Based on this, we develop three algorithms and investigate their performance in the next section:

I. ALG-I: In this algorithm, evaluation is always carried out using the BattBased-DP throughout the proposed DPILNS. That is, in all heuristics accompanied by DPIP (lines 2, 8 and 12 of Algorithm 1), DPIP is replaced by the BattBased-DP. Following the identification of the $\mathcal{S}_{\text {best }}$ at the end of the algorithm (line 23), $\mathcal{S}_{\text {best }}$ is submitted to DPIP for optimal evaluation, and the optimal evaluation is returned.

II. ALG-II: In this algorithm, general evaluation is carried out using the DistBased-DP, and in all heuristics accompanied by DPIP (except for line 2 of Algorithm 1), DPIP is replaced by the DistBased-DP. However, at two main checkpoints, i.e. after that the insertion heuristic returns $\mathcal{S}_{\text {curr }}$ (line 8), and after that the intensification procedure returns $\mathcal{S}_{\text {new }}$, both $\mathcal{S}_{\text {curr }}$ and $\mathcal{S}_{\text {new }}$ are evaluated using DPIP. Two forms of cost, referred to as Cost $t_{\text {opt }}$ and $\operatorname{Cost}_{s u b}$, are therefore maintained for each solution in the course of this algorithm, where the former denotes the cost realised from the optimal evaluation of the solution using DPIP, and the latter refers to the cost realised from the alternative method used for evaluation. While the high level search towards the global optimum is always carried out based on Cost $_{\text {opt }}$ (i.e. lines 9,13,16 and 18 of the algorithm), finding local optima in the insertion and intensification stages is carried out on the basis of $\operatorname{Cost}_{\text {sub }}$.

III. ALG-III: This algorithm is same as ALG-II with the difference that general evaluation is carried out using the BattBased-DP here.

We will apply these three algorithms on a library of EVRPTW-SMBS test instances introduced in the next section, and report on their performance against the optimal solutions and against one another.

\section{Computational results}

In this section, we present the numerical experiments conducted to gain insights on the newly proposed problem of the EVRPTW-SMBS and to evaluate the effectiveness of the proposed solution algorithms. The section begins by introducing the EVRPTW-SMBS test instances, and then applies the proposed mathematical formulation introduced in section 3 on small test instances with up to 25 customers to gain insights on the benefits of using the proposed problem variant as opposed to the solutions based on intra-route recharging. Finally, the performance of the three alternatives of the proposed matheuristic algorithm is evaluated against the optimal and near optimal solutions found to small-sized instances, and by their application on EVRPTW-SMBS instances with 100 customers, and several managerial insights are discussed.

All the experiments were performed on a computer with Intel Core ${ }^{\mathrm{TM}}$ i5 $3.40 \mathrm{GHz}$ processor with $8 \mathrm{~GB}$ RAM. The branch-and-bound solver of CPLEX ${ }^{\mathrm{TM}}$ 12.9.0 was used as the exact solver, and all other algorithms were coded in MATLAB $^{\mathrm{TM}}$. Whenever needed, CPLEX ${ }^{\mathrm{TM}}$ is called from MATLAB ${ }^{\mathrm{TM}}$.

\subsection{Generation of EVRPTW-SMBS test instances}

The EVRPTW-SMBS test instances developed in this paper are created by applying a few modifications on the EVRPTW instances proposed by Schneider et al. (2014). The test problems in Schneider et al. (2014) are developed based on the well-known benchmark instances for the VRPTW proposed by Solomon (1987) which comprise six sets of test problems (C1, R1, RC1, C2, R2, and RC2). Instances in the sets C1 and C2 are with clustered geographical data, instances in R1 and $\mathrm{R} 2$ are generated by a random uniform distribution, and instances in $\mathrm{RC} 1$ and $\mathrm{RC} 2$ are semi-clustered instances that contain a mix of randomly generated data and clusters. Problem sets in the first group (i.e. R1, C1, and RC1) have a short scheduling horizon, whereas the second group instances (i.e. R2, C2, and RC2) have a longer scheduling horizon. To extend these 
instance to their EVRPTW test problems, Schneider et al. (2014) introduce to each one the locations of a set of $21 \mathrm{CSs}$, one at the depot, and the other 20 ones at randomly selected locations, such that every customer can be reached from the depot using at most two different CSs.

Since the detours for visits to CSs and the resulting recharging times in solutions based on intra-route recharging at CSs make it impossible to comply with the customer time windows given in the original Solomon instances, Schneider et al. (2014) generate new time-windows to obtain feasible EVRPTW instances. This, however, is not an issue for solutions based on the use of the BSVs, and as an added value of the proposed variant in this paper, there always exists a feasible solution to the EVRPTW-SMBS if the instance itself is feasible. Therefore, in the proposed EVRPTW-SMBS instances we use the original time windows in the Solomon instances. Moreover, as recharging in CSs is not considered an option in EVRPTW-SMBS, we disregard the location of all CSs; they are, however, retained as potential sites for opening a CS when we are treating the problem as an Electric Location Routing Problem with Time Windows and Partial Recharging (ELRPTW-PR). We are using the same ECV characteristics (i.e. maximum payload, battery capacity, and energy consumption rate per unit distance travelled) used by Schneider et al. (2014) in each instance. BSVs are assumed to have a capacity to carry 5 batteries, a battery capacity twice that of an ECV, and an energy consumption rate per unit distance travelled similar to that of an ECV. The battery swapping service is also assumed to take three time units across all test instances (i.e. $s=3$ ).

As discussed in section 3 of the paper, cost coefficients for the objective function (2) can be calculated using TCO calculation methodologies presented in previous studies. In our computational experiments, we are not using explicit values for the associated costs, and instead, for more generality, reasonable ratios between these cost coefficients are used. Since BSVs are practically ECVs with an on-board facility for battery swapping operations, they incur a relatively higher initial investment and periodically arising costs in comparison with ECVs. We, therefore, assume that the acquisition cost of a BSV is $20 \%$ higher than the acquisition cost of an ECV over a given time period (e.g. a day). Further, operational cost of each unit distance travelled by ECVs or BSVs are calculated to be $2 \%$ of the acquisition cost of an ECV when the distancedependent maintenance costs, energy cost and driver wage are considered. Finally, following Shao et al. (2017), we initially set the cost of a CS $\left(\mathcal{C}_{c}\right)$ at 10 times as much as the acquisition cost of a BSV over the same time period (this is subject to sensitivity analyses later in this section). Therefore, the following cost ratios are used in the models: $\mathcal{C}_{e}=50, \mathcal{C}_{b}=60$, $\mathcal{C}_{d_{e}}=\mathcal{C}_{d_{b}}=1$, and $\mathcal{C}_{c}=600$.

EVRPTW-SMBS instances of sizes 5, 10, 15, 25, and 100 customers are generated. All the test instances developed in this paper along with the details of the reported solutions in this section are available at https://data.kent.ac.uk/id/eprint/105.

\subsection{The added value of the EVRPTW-SMBS}

In this section, the added value of mobile battery swapping is investigated by comparing solutions obtained through the use of BSVs in the design of delivery routes for ECVs with solutions based on intra-route recharging with privately owned CSs. Therefore, each instance of size 5, 10, and 15 customers is solved to optimality (or near optimality) in two different settings; in the first setting the EVRPTW-SMBS formulation described in section 3 of the paper is applied on each instance to find a solution that minimises objective function (2). In the second setting, the problem is treated as an ELRPTW-PR with a generalised cost function involving the opening cost of CSs, the acquisition cost of ECVs and the cost of the total distance travelled by the ECVs. In order to solve the problem in this setting, we have adapted the formulation proposed in Keskin and Çatay (2016) for the EVRPTW with partial recharging, and modified it to incorporate simultaneously the location decisions by assuming the available CSs in each instance are potential recharging sites to open.

The results of the experiments on instances with 5,10 and 15 customers are presented in Table 1 . In this table the headings denote the following: Cost: the cost of each solution, $\mathrm{V}_{\mathrm{E}}$ : total number of ECVs used in the solution; $\mathrm{D}_{\mathrm{E}}$ : total distance travelled by ECVs, C: total number of CSs opened, $\mathrm{V}_{\mathrm{B}}$ : total number of BSVs used in the solution; $\mathrm{D}_{\mathrm{B}}$ : total distance travelled by BSVs, S: total number of battery swaps requested, $\mathrm{V}_{\mathrm{T}}$ : total number of vehicles (ECVS and BSVs) used in the solution, and $\mathrm{D}_{\mathrm{T}}$ : total distance travelled by all vehicles (ECVs and BSVs). The solver is given a maximum of 7200 seconds for each instance, and an italic entry in the table under the 'Cost' heading implies that the reported solution is not optimal and is a solution returned by CPLEX after the termination criterion of 7200 seconds is met. 
Table 1 Comparison of the EVRPTW-SMBS with the ELRPTW-PR $\left(\mathcal{C}_{c}=600\right)$

\begin{tabular}{|c|c|c|c|c|c|c|c|c|c|c|c|c|c|}
\hline \multirow{2}{*}{ No. } & \multirow{2}{*}{ Inst. } & \multicolumn{4}{|c|}{ ELRPTW-PR } & \multicolumn{8}{|c|}{ EVRPTW-SMBS } \\
\hline & & Cost & $\mathrm{V}_{\mathrm{E}}$ & $\mathrm{DE}_{\mathrm{E}}$ & $\mathrm{C}$ & Cost & $V_{E}$ & $\mathrm{D}_{\mathrm{E}}$ & $\mathrm{V}_{\mathrm{B}}$ & Dв & $\mathrm{S}$ & $\mathrm{V}_{\mathrm{T}}$ & $\mathrm{D}_{\mathrm{T}}$ \\
\hline 1 & C101-5 & 546.09 & 5 & 296.09 & 0 & 523.84 & 3 & 254.37 & 1 & 59.464 & 1 & 4 & 313.84 \\
\hline 2 & C103-5 & 315.67 & 3 & 165.67 & 0 & 282.66 & 1 & 152.66 & 1 & 20 & 1 & 2 & 172.66 \\
\hline 3 & C206-5 & 896.10 & 1 & 246.10 & 1 & 472.31 & 1 & 213.78 & 1 & 148.524 & 3 & 2 & 362.31 \\
\hline 4 & C208-5 & 1414.34 & 1 & 164.34 & 2 & 406.45 & 1 & 157.72 & 1 & 138.738 & 2 & 2 & 296.45 \\
\hline 5 & $\mathrm{R} 104-5$ & 911.25 & 3 & 161.25 & 1 & 397.10 & 2 & 157.37 & 1 & 79.732 & 2 & 3 & 237.10 \\
\hline 6 & R105-5 & 932.92 & 3 & 182.92 & 1 & 386.40 & 2 & 165.09 & 1 & 61.312 & 2 & 3 & 226.40 \\
\hline 7 & R202-5 & 813.45 & 1 & 163.45 & 1 & 307.50 & 1 & 126.52 & 1 & 70.98 & 2 & 2 & 197.50 \\
\hline 8 & R203-5 & 1492.71 & 1 & 242.71 & 2 & 506.42 & 2 & 192.89 & 1 & 153.53 & 2 & 3 & 346.42 \\
\hline 9 & $\mathrm{RC} 105-5$ & 988.05 & 3 & 238.05 & 1 & 533.72 & 3 & 237.69 & 1 & 86.023 & 1 & 4 & 323.72 \\
\hline 10 & $\mathrm{RC} 108-5$ & 2266.51 & 3 & 316.51 & 3 & 585.99 & 2 & 245.87 & 1 & 180.115 & 3 & 3 & 425.99 \\
\hline 11 & RC204-5 & 885.16 & 2 & 185.16 & 1 & 379.06 & 1 & 172.43 & 1 & 96.624 & 2 & 2 & 269.06 \\
\hline 12 & RC208-5 & 1417.98 & 1 & 167.98 & 2 & 386.61 & 1 & 170.01 & 1 & 106.603 & 2 & 2 & 276.61 \\
\hline 13 & C101-10 & 1873.18 & 5 & 423.18 & 2 & 719.85 & 3 & 336.64 & 1 & 173.21 & 3 & 4 & 509.85 \\
\hline 14 & C104-10 & 1159.46 & 4 & 359.46 & 1 & 442.87 & 1 & 234.22 & 1 & 98.649 & 3 & 2 & 332.87 \\
\hline 15 & C202-10 & 1024.09 & 3 & 274.09 & 1 & 534.76 & 2 & 249.17 & 1 & 125.589 & 3 & 3 & 374.76 \\
\hline 16 & C205-10 & 1528.28 & 2 & 228.28 & 2 & 518.49 & 2 & 226.01 & 1 & 132.473 & 4 & 3 & 358.49 \\
\hline 17 & R102-10 & 1668.16 & 4 & 268.16 & 2 & 564.57 & 3 & 228.42 & 1 & 126.149 & 3 & 4 & 354.57 \\
\hline 18 & R103-10 & 498.35 & 5 & 248.35 & 0 & 379.81 & 2 & 170.48 & 1 & 49.336 & 2 & 3 & 219.81 \\
\hline 19 & R201-10 & 1080.96 & 4 & 280.96 & 1 & 462.70 & 2 & 234.75 & 1 & 67.951 & 3 & 3 & 302.70 \\
\hline 20 & R203-10 & 1654.25 & 3 & 304.25 & 2 & 475.95 & 1 & 234.49 & 1 & 131.462 & 4 & 2 & 365.95 \\
\hline 21 & $\mathrm{RC} 102-10$ & 1370.29 & 6 & 470.29 & 1 & 852.75 & 4 & 422.11 & 1 & 170.641 & 3 & 5 & 592.75 \\
\hline 22 & $\mathrm{RC} 108-10$ & 1807.04 & 4 & 407.05 & 2 & 757.02 & 3 & 364.76 & 1 & 182.266 & 3 & 4 & 547.02 \\
\hline 23 & RC201-10 & 698.85 & 6 & 398.85 & 0 & 577.54 & 2 & 308.48 & 1 & 109.056 & 4 & 3 & 417.54 \\
\hline 24 & $\mathrm{RC} 205-10$ & 1772.67 & 4 & 372.67 & 2 & 723.13 & 3 & 395.07 & 1 & 118.06 & 3 & 4 & 513.13 \\
\hline 25 & C103-15 & 1381.97 & 6 & 481.97 & 1 & 652.03 & 3 & 347.31 & 1 & 94.72 & 3 & 4 & 442.03 \\
\hline 26 & C106-15 & 1099.54 & 4 & 299.54 & 1 & 603.04 & 3 & 285.20 & 1 & 107.842 & 2 & 4 & 393.04 \\
\hline 27 & C202-15 & 17510.27 & 3 & 400.27 & 2 & 689.64 & 3 & 372.68 & 1 & 106.956 & 4 & 4 & 479.64 \\
\hline 28 & C208-15 & 1589.94 & 2 & 289.94 & 2 & 582.12 & 2 & 275.89 & 1 & 146.224 & 2 & 3 & 422.12 \\
\hline 29 & R102-15 & 1827.49 & 5 & 377.49 & 2 & 645.18 & 3 & 342.39 & 1 & 92.794 & 4 & 4 & 435.18 \\
\hline 30 & R105-15 & 2431.24 & 5 & 381.243 & 3 & 803.36 & 5 & 360.82 & 1 & 132.54 & 4 & 6 & 493.36 \\
\hline 31 & R202-15 & 2366.52 & 3 & 416.523 & 3 & 741.52 & 3 & 385.66 & 1 & 145.852 & 4 & 4 & 531.52 \\
\hline 32 & R209-15 & 1706.45 & 3 & 356.45 & 2 & 562.96 & 2 & 286.47 & 1 & 116.489 & 4 & 3 & 402.96 \\
\hline 33 & RC103-15 & 2523.40 & 5 & 473.40 & 3 & 975.68 & 5 & 491.35 & 1 & 174.332 & 4 & 6 & 665.68 \\
\hline 34 & RC108-15 & 1966.19 & 5 & 516.19 & 2 & 783.27 & 3 & 364.69 & 2 & 148.577 & 4 & 5 & 513.27 \\
\hline 35 & $\mathrm{RC} 202-15$ & 1876.98 & 3 & 526.98 & 2 & 660.09 & 2 & 337.41 & 1 & 162.68 & 4 & 3 & 500.09 \\
\hline 36 & RC204-15 & 1803.51 & 3 & 453.51 & 2 & 602.31 & 2 & 304.47 & 1 & 137.839 & 3 & 3 & 442.31 \\
\hline
\end{tabular}

Table 1 shows that in all cases a solution based on EVRPTW-SMBS is significantly less expensive than a solution based on intra-route recharging. An ELRPTW-PR solution can be up to around 3.9 times more expensive than an EVRPTW-SMBS solution (on average 2.49 times more expensive across all instances). The large cost of the solutions based on intra-route recharging is mainly due to the large cost of opening a CS and in some cases it is less costly to use more ECVs to carry out deliveries than opening a CS and hence a CS is not opened. On the other hand, the table shows that in almost all cases (except instance RC108-15), and regardless of the increasing size of the instances, only one BSV is required to design energy feasible routes for ECVs in EVRPTW-SMBS. The total distance travelled by BSVs is on average $43 \%$ of the distance travelled by ECVs; this percentage, however, tends to decrease as the size of instances increases (e.g. $53 \%, 44 \%$ and $38 \%$ for 5, 10 and 15 customer instances, respectively). As will be also discussed further in the next section, the capacity utilisation rate of BSVs is much better in large instances since in smaller instances there are fewer ECVs requiring battery swapping services (on average $56 \%$ utilisation over all instances in Table 1). In Table 1, note also that the total number of ECVs required and the total distance travelled by them is on average $53 \%$ and $19 \%$ smaller in the case of EVRPTW-SMBS. Even the combined number of BSVs and ECVs required in solutions based on EVRPTW-SMBS is around 5\% smaller than the number of ECVs needed in ELRPTW-PR. There are three cases in Table 1 (instances RC2085, RC205-10, and RC103-15) where the total distance travelled by ECVs is marginally larger in the case of the EVRPTWSMBS. The reason for the larger distance travelled by ECVs in these instances is the trade-off between the cost of the distance travelled by ECVs with the other cost components in the objective function (the acquisition cost of BSVs and 
ECVs, and the cost of the total distance travelled by BSVs). Pure minimisation of the total distance travelled by ECVs can increase the total cost of the solution.

In order to see further the impact of CS siting costs on solutions based on intra-route recharging and their costs against EVRPTW-SMBS solutions, we carry out some sensitivity analysis on $\mathcal{C}_{c}$ and re-solve all test instances using much smaller CS opening costs. Therefore, new CS opening costs with $50 \%, 70 \%$ and $80 \%$ discounts in the initial cost assumed for the CSs are considered (i.e. $\mathcal{C}_{c}=300, \mathcal{C}_{c}=180$, and $\mathcal{C}_{c}=120$, respectively) and all test instances are solved using these new CS opening costs, and the cost of the corresponding ELRPTW-PR is compared with that of the EVRPTW-SMBS given in Table 1. The results of this comparison is given in Table 2. In this table, $\Delta$ is the ratio of the cost of the corresponding ELRPTW-PR solution by the cost of the EVRPTW-SMBS solution.

Table 2 Sensitivity analysis on CS opening cost

\begin{tabular}{|c|c|c|c|c|c|c|c|c|c|c|c|c|c|c|}
\hline \multirow{3}{*}{ No. } & \multirow{3}{*}{ Inst. } & \multicolumn{13}{|c|}{ Various cost coefficients for opening a CS } \\
\hline & & \multirow{2}{*}{$\frac{\mathcal{C}_{c}=600}{\Delta}$} & \multicolumn{4}{|c|}{$\mathcal{C}_{c}=300$} & \multicolumn{4}{|c|}{$\mathcal{C}_{c}=180$} & \multicolumn{4}{|c|}{$\mathcal{C}_{c}=120$} \\
\hline & & & $\Delta$ & $\mathrm{V}_{\mathrm{E}}$ & $\mathrm{D}_{\mathrm{E}}$ & $\mathrm{C}$ & $\Delta$ & $\mathrm{V}_{\mathrm{E}}$ & $\mathrm{D}_{\mathrm{E}}$ & $\mathrm{C}$ & $\Delta$ & $\mathrm{V}_{\mathrm{E}}$ & $\mathrm{D}_{\mathrm{E}}$ & $\mathrm{C}$ \\
\hline 1 & C101-5 & 1.04 & 1.04 & 5 & 296.1 & 0 & 1.04 & 5 & 296.1 & 0 & 1.04 & 5 & 296.1 & 0 \\
\hline 2 & $\mathrm{C} 103-5$ & 1.12 & 1.12 & 3 & 165.7 & 0 & 1.12 & 3 & 165.7 & 0 & 1.12 & 3 & 165.7 & 0 \\
\hline 3 & C206-5 & 1.90 & 1.26 & 1 & 246.1 & 1 & 1.01 & 1 & 246.1 & 1 & 0.88 & 1 & 246.1 & 1 \\
\hline 4 & C208-5 & 3.48 & 2.00 & 1 & 164.3 & 2 & 1.41 & 1 & 164.3 & 2 & 1.12 & 1 & 164.3 & 2 \\
\hline 5 & R104-5 & 2.29 & 1.54 & 3 & 161.3 & 1 & 1.24 & 3 & 161.3 & 1 & 1.09 & 3 & 161.3 & 1 \\
\hline 6 & R105-5 & 2.41 & 1.64 & 3 & 182.9 & 1 & 1.33 & 3 & 182.9 & 1 & 1.17 & 3 & 182.9 & 1 \\
\hline 7 & R202-5 & 2.65 & 1.67 & 1 & 163.4 & 1 & 1.28 & 1 & 163.4 & 1 & 1.08 & 1 & 163.4 & 1 \\
\hline 8 & R203-5 & 2.95 & 1.76 & 1 & 242.7 & 2 & 1.29 & 1 & 242.7 & 2 & 1.05 & 1 & 242.7 & 2 \\
\hline 9 & $\mathrm{RC} 105-5$ & 1.85 & 1.29 & 3 & 238.1 & 1 & 1.06 & 3 & 238.1 & 1 & 0.95 & 3 & 238.1 & 1 \\
\hline 10 & RC108-5 & 3.87 & 2.33 & 3 & 316.5 & 3 & 1.72 & 3 & 316.5 & 3 & 1.41 & 3 & 316.5 & 3 \\
\hline 11 & RC204-5 & 2.34 & 1.54 & 2 & 185.2 & 1 & 1.23 & 2 & 185.2 & 1 & 1.07 & 2 & 185.2 & 1 \\
\hline 12 & RC208-5 & 3.67 & 2.12 & 1 & 168.0 & 2 & 1.50 & 1 & 168.0 & 2 & 1.18 & 1 & 168.0 & 2 \\
\hline 13 & C101-10 & 2.60 & 1.77 & 5 & 423.2 & 2 & 1.44 & 5 & 423.2 & 2 & 1.27 & 5 & 423.2 & 2 \\
\hline 14 & C104-10 & 2.62 & 1.94 & 4 & 359.5 & 1 & 1.67 & 4 & 359.5 & 1 & 1.53 & 4 & 359.5 & 1 \\
\hline 15 & C202-10 & 1.92 & 1.35 & 3 & 274.1 & 1 & 1.13 & 3 & 274.1 & 1 & 1.02 & 3 & 274.1 & 1 \\
\hline 16 & C205-10 & 2.95 & 1.79 & 2 & 228.3 & 2 & 1.33 & 2 & 228.3 & 2 & 1.10 & 2 & 228.3 & 2 \\
\hline 17 & R102-10 & 2.95 & 1.89 & 4 & 268.2 & 2 & 1.47 & 4 & 268.2 & 2 & 1.25 & 4 & 268.2 & 2 \\
\hline 18 & R103-10 & 1.31 & 1.31 & 5 & 248.4 & 0 & 1.31 & 5 & 248.4 & 0 & 1.21 & 3 & 191.0 & 1 \\
\hline 19 & R201-10 & 2.34 & 1.69 & 4 & 281.0 & 1 & 1.43 & 4 & 281.0 & 1 & 1.30 & 4 & 281.0 & 1 \\
\hline 20 & R203-10 & 3.48 & 2.22 & 3 & 304.3 & 2 & 1.71 & 3 & 304.3 & 2 & 1.46 & 2 & 232.7 & 3 \\
\hline 21 & RC102-10 & 1.61 & 1.26 & 6 & 470.3 & 1 & 1.11 & 6 & 470.3 & 1 & 1.04 & 6 & 470.3 & 1 \\
\hline 22 & RC108-10 & 2.39 & 1.59 & 4 & 407.0 & 2 & 1.28 & 4 & 407.0 & 2 & 1.12 & 4 & 407.0 & 2 \\
\hline 23 & RC201-10 & 1.21 & 1.21 & 6 & 398.9 & 0 & 1.21 & 6 & 398.9 & 0 & 1.16 & 3 & 398.9 & 1 \\
\hline 24 & RC205-10 & 2.45 & 1.62 & 4 & 372.7 & 2 & 1.29 & 4 & 372.7 & 2 & 1.12 & 4 & 372.7 & 2 \\
\hline 25 & C103-15 & 2.12 & 1.66 & 6 & 482.0 & 1 & 1.48 & 6 & 482.0 & 1 & 1.37 & 5 & 400.9 & 2 \\
\hline 26 & C106-15 & 1.82 & 1.33 & 4 & 299.5 & 1 & 1.13 & 4 & 299.5 & 1 & 1.03 & 4 & 299.5 & 1 \\
\hline 27 & C202-15 & 2.54 & 1.67 & 3 & 400.3 & 2 & 1.32 & 3 & 400.3 & 2 & 1.15 & 3 & 400.3 & 2 \\
\hline 28 & C208-15 & 2.73 & 1.70 & 2 & 289.9 & 2 & 1.29 & 2 & 289.9 & 2 & 1.08 & 2 & 289.9 & 2 \\
\hline 29 & R102-15 & 2.83 & 1.90 & 5 & 377.5 & 2 & 1.53 & 5 & 377.5 & 2 & 1.34 & 5 & 377.5 & 2 \\
\hline 30 & R105-15 & 3.03 & 1.91 & 5 & 381.2 & 3 & 1.46 & 5 & 381.2 & 3 & 1.23 & 5 & 381.2 & 3 \\
\hline 31 & R202-15 & 3.19 & 1.98 & 3 & 416.5 & 3 & 1.49 & 3 & 416.5 & 3 & 1.25 & 3 & 416.5 & 3 \\
\hline 32 & R209-15 & 3.03 & 1.97 & 3 & 356.4 & 2 & 1.54 & 3 & 356.4 & 2 & 1.33 & 3 & 356.4 & 2 \\
\hline 33 & RC103-15 & 2.59 & 1.66 & 5 & 473.4 & 3 & 1.29 & 5 & 473.4 & 3 & 1.11 & 5 & 473.4 & 3 \\
\hline 34 & RC108-15 & 2.51 & 1.74 & 5 & 516.2 & 2 & 1.44 & 5 & 516.2 & 2 & 1.28 & 5 & 516.2 & 2 \\
\hline 35 & RC202-15 & 2.84 & 1.93 & 3 & 527.0 & 2 & 1.57 & 3 & 527.0 & 2 & 1.39 & 3 & 527.0 & 2 \\
\hline 36 & RC204-15 & 2.99 & 2.00 & 3 & 453.5 & 2 & 1.58 & 1 & 363.1 & 3 & 1.30 & 2 & 320.1 & 3 \\
\hline
\end{tabular}

Table 2 shows that almost all solutions remain unchanged in terms of the number of ECVs required and the total distance travelled by ECVs. The table indicates that with the exception of only two cases which correspond to the scenario where the cost of siting a CS is the smallest (i.e. C206-5 and RC105-5 under $\mathcal{C}_{c}=120$ ), in all other cases the solution based on the use of BSVs is substantially less expensive than the solutions based on intra-route recharging.

We investigate further the benefits of the EVRPTW-SMBS and the performance of the proposed solution algorithms for the problem in the next section. 


\subsection{The performance of the proposed algorithms}

In this section, we first compare the solutions obtained using the three different variants of the DP-ILNS discussed in section 4 (i.e. ALG-I, ALG-II and ALG-III) with the optimal (or near optimal) solutions found for small EVRPTW-SMBS instances discussed in the previous section. To generate a benchmark of a larger size, we have also put EVRPTW-SMBS instances with 25 customers into CPLEX and found optimal or near optimal solutions to some of the 56 instances considered within an allowed CPU time of 7200 seconds (see Appendix B, Table B.1). Finally, the performance of the proposed algorithms in solving instances with 100 customers is analysed. All algorithms were run 10 times on each instance, and the best result is returned. Moreover, following a preliminary set of empirical analyses, required parameters for all algorithms were fine-tuned as follows:

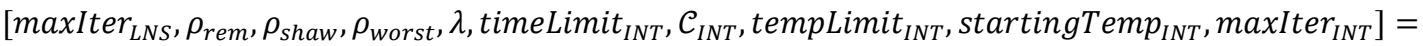
$[30 n, 0.8,6,3, k / 75,5,0.92,300,500,4 k]$ (where $k$ is the number of routes in $\mathcal{S}_{\text {curr }}$ ).

Furthermore, in DPIP a maximum labels set size of 1000 is considered and a maximum CPU time of 3 seconds is allocated to the solver for LSP. While the chosen settings generally work well on the considered test problems, no claim is made that our choice of parameter values is the best possible.

Table 3 presents the aggregated results for instance groups with 5, 10, 15 and 25 customers. The table headings have the same meaning as in Table 1, but the entries for each algorithm under each heading are based on the average over all instances in the corresponding group. In this table and all other tables, $\mathrm{t}(\mathrm{s})$ is the average computing time (CPU) in seconds over ten runs. Also, the 'Gap' column reports the average gap with the solution returned by CPLEX. Table 3 clearly shows that, ALG-I which relies only on the use of the DPIP in the post-optimisation stage, provides a less favourable performance compared to the other two algorithms, i.e. ALG-II and ALG-III. On the other hand, ALG-II and ALG-III which are calling DPIP at a higher frequency over the course of the algorithm (at the cost of a higher computational cost), have generated results that are very closely comparable.

Table 3 Aggregate results of solving small instances using the proposed algorithms

\begin{tabular}{llllllllllll}
\hline Inst. group & Alg. & Cost & $\mathrm{V}_{\mathrm{E}}$ & $\mathrm{D}_{\mathrm{E}}$ & $\mathrm{V}_{\mathrm{B}}$ & $\mathrm{D}_{\mathrm{B}}$ & $\mathrm{S}$ & $\mathrm{V}_{\mathrm{T}}$ & $\mathrm{D}_{\mathrm{T}}$ & $\mathrm{Gap}$ & $\mathrm{t}(\mathrm{s})$ \\
\hline \multirow{5}{*}{ 5-cust. } & ALG-I & 448.66 & 1.67 & 184.19 & 1.17 & 111.13 & 2.08 & 2.83 & 295.32 & 0.04 & 0.02 \\
& ALG-II & 430.97 & 1.67 & 187.50 & 1.00 & 100.14 & 1.92 & 2.67 & 287.64 & 0.00 & 0.05 \\
& ALG-III & 430.97 & 1.67 & 187.50 & 1.00 & 100.14 & 1.92 & 2.67 & 287.64 & 0.00 & 0.04 \\
& & & & & & & & & & & \\
10-cust. & ALG-I & 617.99 & 2.17 & 273.74 & 1.50 & 145.92 & 3.50 & 3.67 & 419.65 & 0.05 & 0.06 \\
& ALG-II & 590.76 & 2.33 & 283.71 & 1.08 & 125.38 & 3.17 & 3.42 & 409.09 & 0.01 & 0.27 \\
& ALG-III & 585.64 & 2.25 & 280.38 & 1.08 & 127.76 & 3.33 & 3.33 & 408.14 & 0.00 & 0.17 \\
& ALG-I & 706.96 & 2.58 & 331.27 & 1.50 & 156.52 & 4.08 & 4.08 & 487.79 & 0.02 & 1.08 \\
25-cust. & ALG-II & 690.90 & 2.67 & 335.88 & 1.33 & 141.68 & 4.00 & 4.00 & 477.57 & 0.00 & 2.45 \\
& ALG-III & 692.91 & 2.67 & 337.92 & 1.33 & 141.66 & 3.83 & 4.00 & 479.58 & 0.00 & 2.65 \\
& ALG-I & 844.85 & 3.71 & 462.33 & 1.29 & 119.66 & 3.46 & 5.00 & 581.99 & 0.02 & 34.56 \\
& ALG-II & 822.84 & 3.84 & 467.83 & 1.07 & 98.76 & 3.14 & 4.91 & 566.59 & 0.00 & 74.49 \\
& ALG-III & 828.52 & 3.82 & 468.71 & 1.14 & 100.17 & 3.25 & 4.96 & 568.88 & 0.00 & 67.41 \\
& & & & & & & & & & & \\
Avg. all & ALG-I & 745.59 & 3.10 & 384.36 & 1.33 & 126.78 & 3.37 & 4.42 & 511.14 & 0.03 & 21.19 \\
& ALG-II & 724.25 & 3.21 & 390.04 & 1.10 & 108.01 & 3.10 & 4.30 & 498.05 & 0.00 & 45.71 \\
& ALG-III & 727.30 & 3.18 & 390.41 & 1.14 & 109.18 & 3.16 & 4.33 & 499.58 & 0.00 & 41.40 \\
\hline
\end{tabular}

A more detailed comparison of the results found by these algorithms against the optimal solutions is presented in Appendix B, in Tables B.2 and B. 3 for small sized instances and instances with 25 customers, respectively. Overall, these tables show that solutions returned by ALG-I can have an optimality gap of as large as 18\%, but ALG-II and ALG-III can very well approximate the optimal solutions to small sized instances and the available optimal solutions to 25-customer instances. The solutions returned by ALG-II and ALG-III matches exactly with the optimal solutions in most of the cases. The relatively less favourable performance of ALG-I is an indication of the strong presence of the interdependence problem in the case of the EVRPTW-SMBS, which cannot be simply overcome by treating the routes in two completely separate levels.

In order to investigate further the performance of the proposed algorithms in solving more practically sized problem instances, the three algorithms have been further applied on the proposed test problems with 100 customers and the results are presented in Table 4 and Table 5. In Table 4, the aggregated results for instance groups of C1, C2, R1, R2, RC1 and 
RC2 are presented under the same headings as before. The 'Gap' column here reports the average gap with the best solutions found for instances in each group. In line with the previous results from small test instances, the table clearly shows that ALG-I is outperformed by the other two algorithms across almost all instance groups. The less favourable performance of ALG-I is particularly highlighted in the case of RC2 instances which are semi-clustered instances containing a mix of randomly generated data and clusters with a relatively longer planning horizon. On the other hand, ALG-III demonstrates to be delivering the best performance, overall. The average gap of the solutions found by ALG-III with the best-found solutions is zero in the case of most of the instance groups (i.e. C1, C2, R1, and RC1). Finally, algorithm ALG-II delivers a middle performance, being closer to ALG-I in most cases than to ALG-III. The performance of the algorithm is particularly worse in case of R1 and RC1 instances, and it is not generally outperforming ALG-III in any of the instance groups. Furthermore, ALG-III is faster than ALG-II and is therefore a prime choice for the problem.

Note also in Table 4 the total number of BSVs required and the total distance travelled by them in relation with the total distance driven by ECVs. The number of the required BSVs is not substantially larger than the number of BSVs in small sized instances and only a few BSVs are sufficient to support the routes of several ECVs. The ratio of the BSV distance to ECV distance is also considerably smaller than small sized instances. These insights are briefly discussed at the end of this section.

Table 4 Aggregate results of solving instances with 100 customers using the proposed algorithms

\begin{tabular}{llllllllllll}
\hline Inst. group & Alg. & Cost. & $\mathrm{V}_{\mathrm{E}}$ & $\mathrm{D}_{\mathrm{E}}$ & $\mathrm{V}_{\mathrm{B}}$ & $\mathrm{D}_{\mathrm{B}}$ & $\mathrm{S}$ & $\mathrm{V}_{\mathrm{T}}$ & $\mathrm{D}_{\mathrm{T}}$ & $\mathrm{Gap}$ & $\mathrm{t}(\mathrm{s})$ \\
\hline \multirow{4}{*}{ C1 } & ALG-I & 1800.90 & 10.44 & 883.19 & 2.11 & 268.82 & 5.56 & 12.56 & 1152.00 & 0.04 & 198.88 \\
& ALG-II & 1778.90 & 10.22 & 881.47 & 2.11 & 259.68 & 5.78 & 12.33 & 1141.10 & 0.02 & 519.21 \\
& ALG-III & 1742.50 & 9.89 & 858.43 & 2.11 & 262.93 & 5.89 & 12.00 & 1121.40 & 0.00 & 339.84 \\
C2 & ALG-I & 1114.50 & 4.13 & 695.69 & 1.13 & 145.08 & 4.13 & 5.25 & 840.77 & 0.05 & 126.81 \\
& ALG-II & 1073.60 & 4.13 & 670.38 & 1.00 & 136.95 & 3.75 & 5.13 & 807.33 & 0.01 & 336.54 \\
& ALG-III & 1060.00 & 4.13 & 653.15 & 1.00 & 140.56 & 3.88 & 5.13 & 793.71 & 0.00 & 276.68 \\
R1 & ALG-I & 2676.20 & 16.83 & 1312.20 & 3.25 & 327.29 & 12.50 & 20.08 & 1639.50 & 0.05 & 276.73 \\
& ALG-II & 2691.10 & 16.67 & 1335.70 & 3.33 & 322.04 & 12.75 & 20.00 & 1657.70 & 0.06 & 507.69 \\
& ALG-III & 2558.80 & 15.00 & 1269.50 & 3.58 & 324.29 & 13.67 & 18.58 & 1593.80 & 0.00 & 502.42 \\
R2 & ALG-I & 1367.13 & 4.64 & 1030.39 & 0.91 & 50.38 & 2.36 & 5.55 & 1080.77 & 0.05 & 122.03 \\
& ALG-II & 1319.74 & 4.64 & 983.06 & 0.91 & 50.32 & 2.36 & 5.55 & 1033.38 & 0.01 & 290.48 \\
& ALG-III & 1318.82 & 4.64 & 982.08 & 0.91 & 50.38 & 2.27 & 5.55 & 1032.46 & 0.01 & 204.90 \\
RC1 & ALG-I & 2864.20 & 15.50 & 1499.70 & 3.50 & 379.46 & 12.50 & 19.00 & 1879.20 & 0.06 & 268.89 \\
& ALG-II & 2841.90 & 15.50 & 1513.20 & 3.13 & 366.19 & 12.00 & 18.63 & 1879.40 & 0.05 & 666.93 \\
& ALG-III & 2708.90 & 14.25 & 1438.00 & 3.13 & 370.87 & 11.88 & 17.38 & 1808.90 & 0.00 & 595.74 \\
& & & & & & & & & & & \\
RC2 & ALG-I & 1663.40 & 4.88 & 1243.00 & 1.25 & 101.68 & 3.63 & 6.13 & 1344.70 & 0.13 & 141.57 \\
& ALG-II & 1524.30 & 4.88 & 1137.30 & 1.00 & 83.17 & 3.13 & 5.88 & 1220.50 & 0.03 & 381.87 \\
& ALG-III & 1490.40 & 4.88 & 1104.40 & 1.00 & 82.19 & 2.50 & 5.88 & 1186.60 & 0.01 & 321.36 \\
& & & & & & & & & & \\
Avg. all & ALG-I & 1937.46 & 9.70 & 1116.73 & 2.05 & 212.69 & 6.93 & 11.75 & 1329.42 & 0.06 & 191.98 \\
& ALG-II & 1898.89 & 9.63 & 1095.40 & 1.96 & 204.38 & 6.82 & 11.59 & 1299.79 & 0.03 & 447.20 \\
& ALG-III & 1838.72 & 9.04 & 1059.42 & 2.02 & 206.44 & 6.93 & 11.05 & 1265.86 & 0.00 & 373.06 \\
\hline \multirow{6}{*}{} & & & & & & & & & & &
\end{tabular}

The details of the performance of the algorithms in case of each of the 56 test instances and with respect to the generalised cost of the solutions obtained and runtime in seconds are given in Table 5. Solutions in Bold show the bestfound solution for each instance. The table shows that ALG-I, ALG-II and ALG-III contribute to the identification of 6, 23 , and 49 solutions out of the best-found solutions, respectively. Note that the computational cost of ALG-II is partially larger than ALG-III due to the relatively larger number of endpoint labels generated by the DistBased-DP used in ALG-II, compared with the BattBased-DP used within ALG-III. Indeed, in BattBased-DP, due to the extra domination rule used based on the total number of batteries required, the set of end-point labels is almost always smaller, and hence DPIP is run faster. 
Table 5 Cost and runtime of solutions returned by the proposed algorithms for instances with 100 customers

\begin{tabular}{|c|c|c|c|c|c|c|c|}
\hline \multirow{2}{*}{ No. } & \multirow{2}{*}{ Inst. } & \multicolumn{2}{|l|}{ ALG-I } & \multicolumn{2}{|l|}{ ALG-II } & \multicolumn{2}{|l|}{ ALG-II } \\
\hline & & Cost & $\mathrm{t}(\mathrm{s})$ & Cost & $\mathrm{t}(\mathrm{s})$ & Cost & $t(s)$ \\
\hline 1 & C101-100 & 1791.11 & 189.15 & 1784.80 & 565.45 & 1714.44 & 532.57 \\
\hline 2 & C102-100 & 1792.55 & 226.81 & 1714.44 & 677.86 & 1714.44 & 232.65 \\
\hline 3 & C103-100 & 1713.56 & 176.06 & 1713.56 & 654.76 & 1720.40 & 355.44 \\
\hline 4 & C104-100 & 2077.47 & 212.66 & 2111.27 & 232.56 & 1954.51 & 353.31 \\
\hline 5 & C105-100 & 1711.92 & 174.26 & 1711.92 & 636.88 & 1711.92 & 605.54 \\
\hline 6 & C106-100 & 1714.44 & 171.35 & 1774.60 & 301.92 & 1714.44 & 389.16 \\
\hline 7 & C107-100 & 1793.70 & 232.18 & 1691.73 & 219.17 & 1711.92 & 197.44 \\
\hline 8 & C108-100 & 1788.88 & 184.93 & 1796.03 & 850.80 & 1711.92 & 227.03 \\
\hline 9 & C109-100 & 1824.47 & 222.54 & 1711.92 & 533.49 & 1728.23 & 165.44 \\
\hline 10 & C201-100 & 1047.98 & 95.33 & 1050.26 & 399.11 & 1028.94 & 435.46 \\
\hline 11 & C202-100 & 1051.66 & 141.17 & 1032.37 & 439.47 & 1032.37 & 380.89 \\
\hline 12 & C203-100 & 1096.37 & 120.95 & 1089.34 & 434.16 & 1085.67 & 241.80 \\
\hline 13 & C204-100 & 1237.83 & 104.98 & 1095.98 & 263.58 & 1095.98 & 216.48 \\
\hline 14 & C205-100 & 1075.40 & 183.05 & 1064.19 & 280.14 & 1061.83 & 281.00 \\
\hline 15 & C206-100 & 1082.32 & 131.42 & 1107.55 & 508.15 & 1075.81 & 282.03 \\
\hline 16 & C207-100 & 1105.14 & 140.03 & 1085.98 & 150.19 & 1064.88 & 170.66 \\
\hline 17 & C208-100 & 1219.50 & 97.52 & 1062.98 & 217.50 & 1034.16 & 205.08 \\
\hline 18 & R101-100 & 3664.11 & 312.50 & 3468.73 & 601.64 & 3417.16 & 775.44 \\
\hline 19 & R102-100 & 3253.19 & 460.26 & 3253.19 & 677.06 & 3291.76 & 672.83 \\
\hline 20 & R103-100 & 2548.63 & 370.45 & 2640.67 & 477.83 & 2535.63 & 456.98 \\
\hline 21 & R104-100 & 2272.69 & 201.35 & 2231.69 & 387.42 & 2231.69 & 590.14 \\
\hline 22 & R105-100 & 2916.17 & 273.22 & 2937.61 & 325.54 & 2842.92 & 247.02 \\
\hline 23 & R106-100 & 2686.83 & 202.04 & 2804.09 & 553.83 & 2569.08 & 607.48 \\
\hline 24 & R107-100 & 2557.16 & 310.93 & 2485.65 & 412.42 & 2403.36 & 408.38 \\
\hline 25 & R108-100 & 2148.42 & 271.55 & 2296.56 & 290.46 & 2066.61 & 545.31 \\
\hline 26 & R109-100 & 2879.29 & 189.13 & 2879.29 & 604.60 & 2540.60 & 257.84 \\
\hline 27 & R110-100 & 2442.14 & 226.79 & 2539.78 & 228.04 & 2399.10 & 524.25 \\
\hline 28 & R111-100 & 2521.45 & 302.72 & 2521.45 & 814.99 & 2280.18 & 656.40 \\
\hline 29 & R112-100 & 2223.90 & 199.76 & 2234.17 & 718.46 & 2127.51 & 286.95 \\
\hline 30 & R201-100 & 1710.68 & 202.11 & 1704.93 & 318.71 & 1704.93 & 222.28 \\
\hline 31 & R202-100 & 1479.65 & 111.43 & 1441.24 & 113.63 & 1441.24 & 273.36 \\
\hline 32 & R203-100 & 1574.90 & 113.58 & 1307.93 & 159.90 & 1307.93 & 340.30 \\
\hline 33 & R204-100 & 1056.81 & 132.36 & 1103.85 & 411.35 & 1027.33 & 180.80 \\
\hline 34 & R205-100 & 1529.92 & 162.71 & 1467.31 & 480.39 & 1383.82 & 273.17 \\
\hline 35 & R206-100 & 1470.32 & 91.73 & 1305.78 & 342.96 & 1305.78 & 151.93 \\
\hline 36 & R207-100 & 1240.21 & 107.79 & 1240.21 & 197.91 & 1240.21 & 280.48 \\
\hline 37 & R208-100 & 1047.71 & 95.22 & 1025.11 & 125.37 & 1025.11 & 95.55 \\
\hline 38 & R209-100 & 1343.14 & 87.82 & 1343.14 & 251.00 & 1343.14 & 138.23 \\
\hline 39 & R210-100 & 1335.03 & 159.96 & 1327.62 & 504.90 & 1506.07 & 182.97 \\
\hline 40 & R211-100 & 1250.04 & 77.65 & 1250.04 & 289.18 & 1221.45 & 114.79 \\
\hline 41 & RC101-100 & 3370.54 & 262.23 & 3385.54 & 1021.37 & 3202.73 & 683.41 \\
\hline 42 & RC102-100 & 3158.49 & 384.92 & 3054.29 & 754.26 & 2825.85 & 1123.68 \\
\hline 43 & RC103-100 & 2634.44 & 211.03 & 2590.92 & 372.73 & 2590.92 & 591.65 \\
\hline 44 & RC104-100 & 2349.36 & 195.58 & 2327.39 & 486.76 & 2327.39 & 429.36 \\
\hline 45 & $\mathrm{RC} 105-100$ & 3246.54 & 243.09 & 3284.80 & 1008.46 & 3143.37 & 324.06 \\
\hline 46 & RC106-100 & 2929.37 & 390.41 & 2823.92 & 936.73 & 2682.90 & 527.03 \\
\hline 47 & RC107-100 & 2538.58 & 269.02 & 2581.71 & 297.36 & 2492.25 & 401.76 \\
\hline 48 & RC108-100 & 2686.35 & 194.81 & 2686.35 & 457.78 & 2405.47 & 684.97 \\
\hline 49 & RC201-100 & 1904.68 & 253.45 & 1862.73 & 546.27 & 1787.23 & 381.47 \\
\hline 50 & RC202-100 & 1797.20 & 121.02 & 1533.68 & 558.50 & 1533.68 & 448.34 \\
\hline 51 & RC203-100 & 1566.45 & 96.04 & 1393.35 & 166.47 & 1396.03 & 416.45 \\
\hline 52 & RC204-100 & 1512.93 & 102.28 & 1205.84 & 386.05 & 1329.25 & 214.07 \\
\hline 53 & $\mathrm{RC} 205-100$ & 1975.40 & 126.75 & 1826.62 & 525.44 & 1754.34 & 373.93 \\
\hline 54 & RC206-100 & 1713.15 & 179.53 & 1713.90 & 655.17 & 1496.26 & 270.06 \\
\hline 55 & RC207-100 & 1456.78 & 168.48 & 1451.20 & 109.72 & 1419.23 & 217.39 \\
\hline 56 & RC208-100 & 1380.73 & 85.02 & 1206.79 & 107.34 & 1206.79 & 249.13 \\
\hline Avg. & & 1953.47 & 193.07 & 1913.35 & 447.85 & 1853.47 & 376.56 \\
\hline
\end{tabular}


For representation, the ECV and BSV routes of two randomly selected instances from groups $\mathrm{C}$ and $\mathrm{R}$ are presented in Figure 6. We remind that customers in group $\mathrm{C}$ instances are clustered, whereas they are randomly distributed in group $\mathrm{R}$ instances.
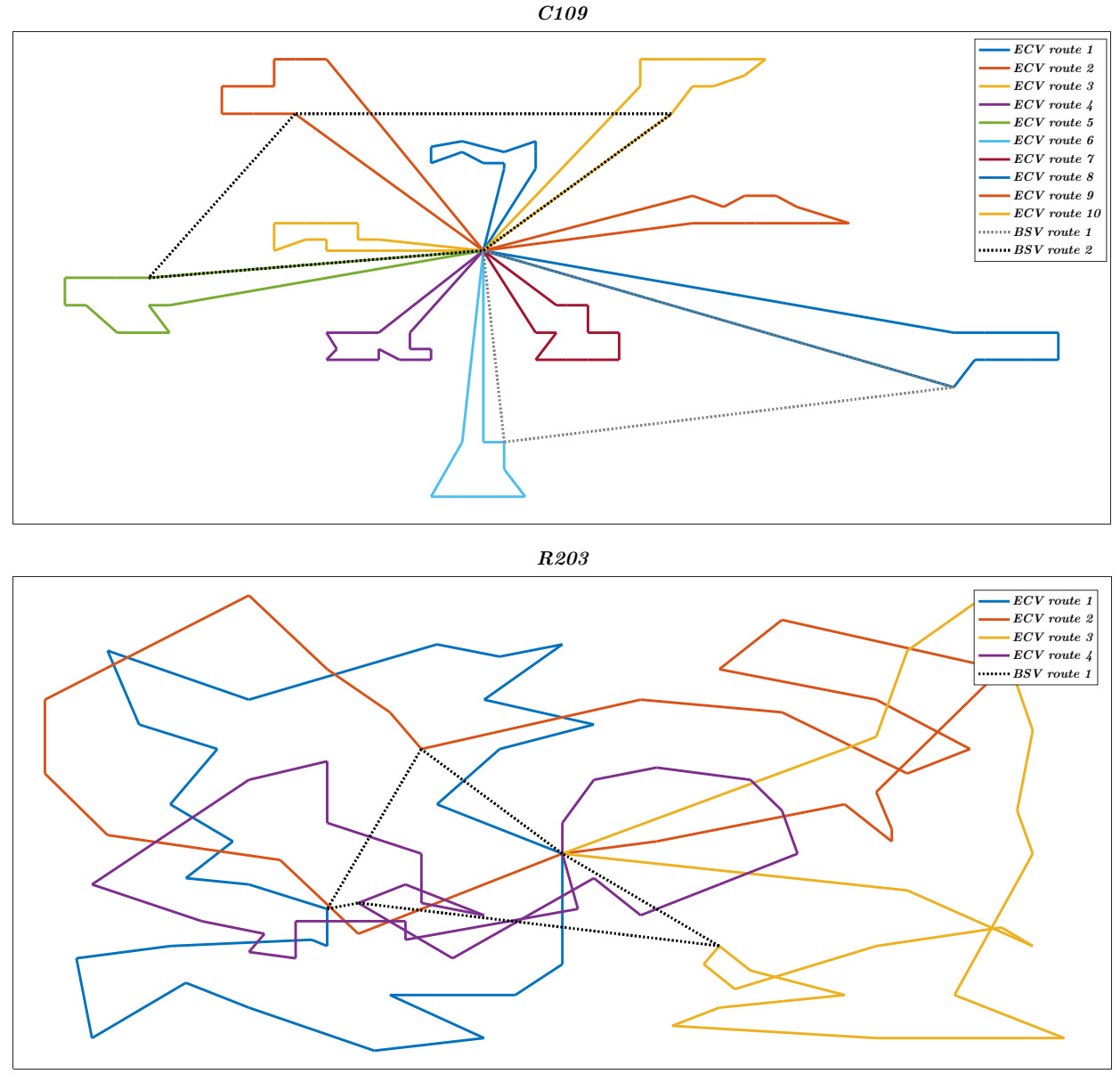

Figure 6 Solution to EVRPTW-SMBS instances with 100 customers

In Figure 6, the solid lines represent ECV routes and the dotted lines are BSV routes. In instance C109, 10 ECVs drive a total distance of 828.94, and 2 BSVs a distance of 262.98 to deliver 5 battery swapping services to 5 different ECV routes on-the-fly. In instance R203, on the other hand, 4 ECVs drive a total distance of 957.71, and 1 BSV a distance of 90.22 to deliver 4 battery swapping services to 4 different ECV routes. The figure clearly shows the complexity and the added value of the EVRPTW-SMBS, where support from BSVs are relied on to design delivery routes.

Finally, to gain some managerial insights from the experiments carried out in this section, in Figure 7.a, we are illustrating the average number of BSVs required compared with the average number of ECVs required for instances grouped based on their sizes, Figure 7.b shows the average distance travelled by BSVs compared with the average distance travelled by ECVs, and Figure 7.c illustrates the utilisation rate of BSVs. To calculate the utilisation rate, given that each BSV has a capacity of 5 batteries, the number of battery swaps delivered in the solution is divided by 5 times the number of BSVs acquired. 


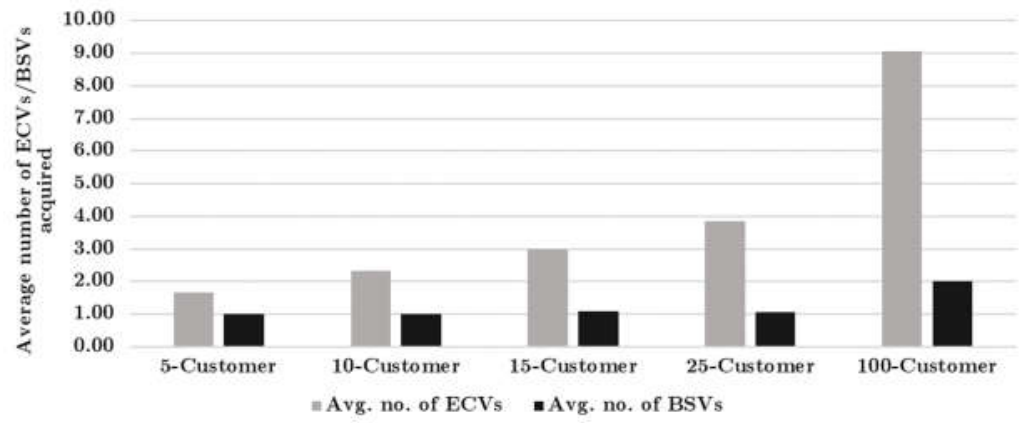

a.

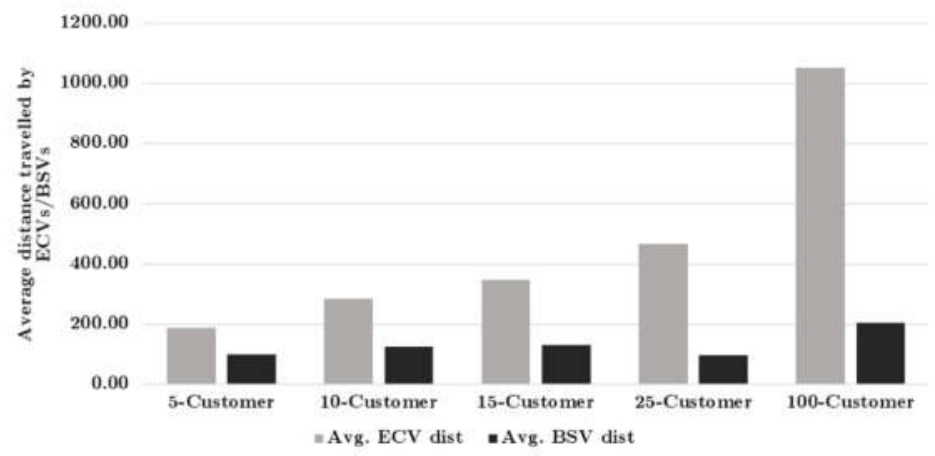

b.

=Avg. ECV dist =Avg. BSV dist

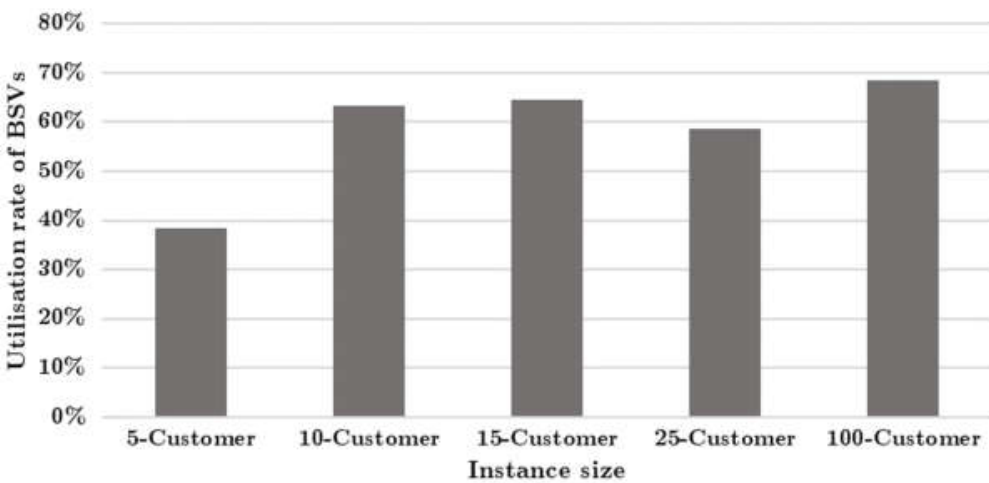

Figure 7 (a) average number of BSVs acquired compared with average number of ECVs acquired, (b) average BSV distance compared with the average ECV distance travelled, (c) utilisation rate of BSVs

The main implication of Figure 7.a may be that with the increasing size of the problem, the number of BSVs required does not increase significantly. As this figure illustrates, for a problem with 100 customers, only 2 BSVs are on average required, and each required BSV can in turn support the route of around 4.5 ECVs. Figure 7.b and Figure 7.c, on the other hand, are on the utilisation rate of the acquired BSVs. Expectedly, the utilisation rate of BSVs improves in larger problem instances. This may have some implications regarding the decision on whether to own a BSV fleet or to hire them from a third-party provider depending on the size of the logistic network. It can be an interesting analytical task of further research.

\section{Discussion and conclusion}

To address the issue of "range anxiety" in goods distribution using ECVs, in this paper a paradigm shift in EVRPTWs was proposed by exploiting relevant technological developments that make mobile battery swapping possible. The problem class of the EVRPTW-SMBS in which an ECV can request a battery swapping service from a BSV on-the-fly was introduced and formulated. In the EVRPTW-SMBS, routing is carried out in two interdependent levels for the ECVs operating delivery routes and for the BSVs. A BSV is able to serve several ECVs in one BSV route, and an ECV can benefit from multiple battery swaps along its route. The use of BSVs as facilitators in goods distribution using ECVs can bring in 
substantial flexibility and cost savings and presents potentials for using ECVs in inter-urban, as well as intra-urban, freight distribution. In addition to their extra flexibility and reliability, solutions based on the use of BSVs can be cost saving compared to solutions based on intra-route recharging from fixed charging stations. Our findings suggest that only a small number of BSVs are required to support the routes of several ECVs delivering to a large number of customers.

The EVRPTW-SMBS, however, is a very difficult problem to tackle due to the interdependence problem and the spatio-temporal synchronisation requirements between ECV and BSV routes. To address these complications, the paper developed a methodology for the exact evaluation of an EVRPTW-SMBS solution using a two stage procedure combining a DP and an IP (the DPIP), and placed the proposed DPIP at the heart of an ILNS heuristic. New benchmark instances for the EVRPTW-SMBS were derived from existing EVRPTW test instances and several numerical experiments were conducted to demonstrate the added value of BSVs and the effectiveness of the developed algorithms. The benefits of the EVRPTW-SMBS were demonstrated in comparison with solutions based on intra-route recharging and it was shown that due to the significant cost of opening a CS, a solution based on intra-route recharging can be up to around 3.9 times more expensive than an EVRPTW-SMBS solution. The efficiency of the proposed matheuristics was demonstrated against the available optimal (or near optimal) solutions and through their application on large sized EVRPTW-SMBS test instances with 100 customers. The numerical experiments demonstrated that in particular one of the algorithms which is based on a specific lexicographical decomposition of the DPIP is able to provide good near-optimal solutions to the EVRPTW-SMBS.

There are multiple future research opportunities in using BSVs in the design of delivery routes for ECVs. Future research may investigate the situations when BSVs are hired from a third-party company with a separate depot, or when BSVs are used as emergency aids in a logistic design based on intra-route recharging. The consideration of a BSV fleet that is a mix of electric, hybrid and internal combustion engine vehicles is another interesting aspect to explore further; use of BSVs with a larger driving range can increase significantly the autonomy of running ECVs by providing support more flexibly and hence allowing the use of ECVs for long-distance deliveries as well as last-mile deliveries. The use of BSVs to deliver small packages along their original responsibility of providing battery swapping service to other ECVs may be also another interesting idea to research. To reuse the expensive resources in the fleet, multi-trip planning of ECVs and BSVs can help reduce vehicle acquisition costs and can be an important future line of research. The results of this paper imply that there is a significant trade-off between the potential key performance indicators in a logistic system based on the use of BSVs and multi-objective optimisation of the EVRPTW-SMBS can provide a useful insight into the trade-offs among the major cost elements of a delivery system that is to exploit the opportunity of mobile battery swapping. More realistic variants of the proposed problem may incorporate stochastic and uncertain travel times and service times. These considerations make it yet more difficult to tackle the temporal synchronisation requirements. As was discussed in section 3.2 of the paper, the consideration of mobile battery recharging vans is also an interesting and promising line of research. The modelling and solution approach presented in this paper may also stimulate new research within the growing literature on truck-drone routing systems. Finally, the development of efficient solution algorithms that can tackle the interdependence problem in the EVRPTW-SMBS is an important future task.

\section{Acknowledgement}

The authors are grateful to the reviewers and the associate editor James F. Campbell for their very constructive comments that have helped us to improve the presentation of this paper.

\section{Appendices}

\section{Appendix A. List of the notation}

\begin{tabular}{ll}
\hline Notation & Definition \\
\hline$G$ & The complete graph on which EVRPTW-SMBS is defined. \\
$N$ & Set of network nodes in $G$. \\
$A$ & Set of directed arcs in $G$. \\
$\sigma$ & A dummy copy of the depot node $\{0\}$ (referred to as the final depot). \\
$D$ & Set of the depot $\{0\}$ and its dummy copy $\{\sigma\}$. \\
$C$ & Set of customer nodes. \\
$n$ & Number of customers in $C$. \\
$q_{i}$ & Demand requested by customer $i \in C$. \\
{$\left[e_{i}, l_{i}\right]$} & Hard time window of customer $i \in C$.
\end{tabular}




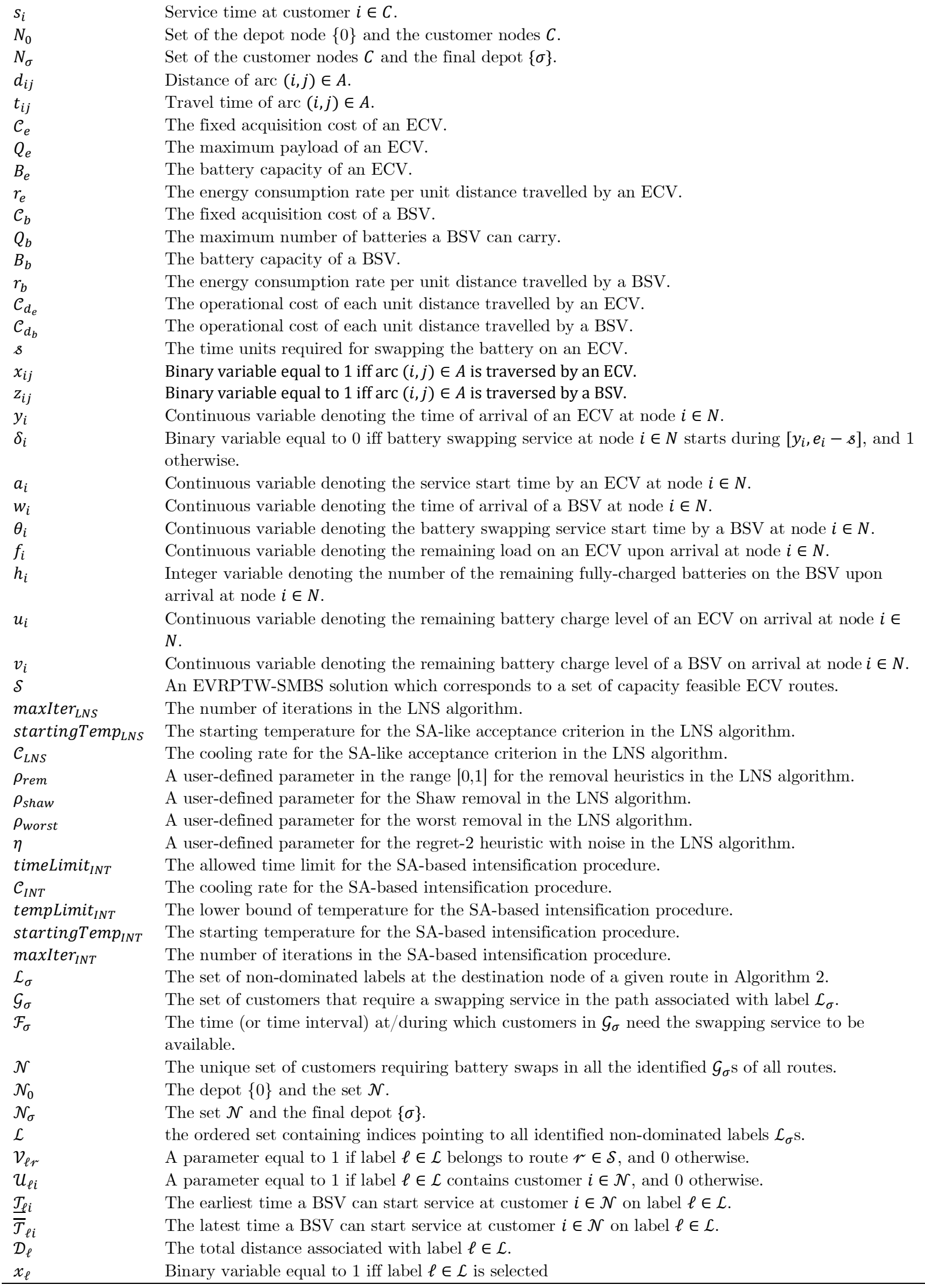




\section{Appendix B. Detailed results of experiments}

Table B.1 CPLEX results for the EVRPTW-SMBS instances with 25 customers

\begin{tabular}{|c|c|c|c|c|c|c|c|c|c|}
\hline No. & Inst. & Cost & $V_{E}$ & $\mathrm{DE}_{\mathrm{E}}$ & $V_{B}$ & $\mathrm{DB}_{\mathrm{B}}$ & $\mathrm{S}$ & $\mathrm{V}_{\mathrm{T}}$ & $\mathrm{D}_{\mathrm{T}}$ \\
\hline 1 & C101-25 & 1175.42 & 5 & 621.10 & 2 & 184.315 & 5 & 7 & 805.42 \\
\hline 2 & C102-25 & $1045.82^{*}$ & 6 & 532.19 & 1 & 153.631 & 4 & 7 & 685.82 \\
\hline 3 & C103-25 & - & - & - & - & - & 0 & 0 & 0.00 \\
\hline 4 & C104-25 & 859.85 & 4 & 445.08 & 1 & 154.771 & 4 & 5 & 599.85 \\
\hline 5 & C105-25 & 1032.38 & 5 & 543.97 & 2 & 118.407 & 6 & 7 & 662.38 \\
\hline 6 & C106-25 & 1090.57 & 6 & 535.77 & 1 & 194.797 & 4 & 7 & 730.57 \\
\hline 7 & C107-25 & 960.44 & 4 & 528.61 & 2 & 111.826 & 7 & 6 & 640.44 \\
\hline 8 & C108-25 & - & - & - & - & - & - & - & - \\
\hline 9 & C109-25 & 875.38 & 4 & 475.32 & 1 & 140.056 & 4 & 5 & 615.38 \\
\hline 10 & C201-25 & 687.80 & 3 & 395.02 & 1 & 82.781 & 2 & 4 & 477.80 \\
\hline 11 & C202-25 & 687.39 & 3 & 423.57 & 1 & 53.815 & 1 & 4 & 477.39 \\
\hline 12 & C203-25 & 738.61 & 5 & 488.61 & 0 & 0.000 & 0 & 5 & 488.61 \\
\hline 13 & C204-25 & 557.76 & 2 & 358.16 & 1 & 39.605 & 2 & 3 & 397.76 \\
\hline 14 & C205-25 & 636.22 & 4 & 436.22 & 0 & 0.000 & 0 & 4 & 436.22 \\
\hline 15 & C206-25 & 680.04 & 3 & 388.85 & 1 & 81.185 & 2 & 4 & 470.04 \\
\hline 16 & C207-25 & 667.74 & 3 & 387.03 & 1 & 70.710 & 1 & 4 & 457.74 \\
\hline 17 & C208-25 & - & - & - & - & - & - & - & - \\
\hline 18 & R101-25 & 1330.84 & 8 & 634.91 & 2 & 175.935 & 8 & 10 & 810.85 \\
\hline 19 & R102-25 & 1207.84 & 8 & 557.11 & 2 & 130.736 & 6 & 10 & 687.84 \\
\hline 20 & R103-25 & - & - & - & - & - & - & - & - \\
\hline 21 & R104-25 & 739.50 & 4 & 359.98 & 1 & 119.512 & 3 & 5 & 479.50 \\
\hline 22 & R105-25 & 1144.43 & 5 & 538.99 & 2 & 235.434 & 7 & 7 & 774.43 \\
\hline 23 & R106-25 & 996.51 & 5 & 472.53 & 2 & 153.981 & 5 & 7 & 626.51 \\
\hline 24 & R107-25 & 892.55 & 4 & 398.72 & 2 & 173.832 & 5 & 6 & 572.55 \\
\hline 25 & R108-25 & 920.96 & 4 & 411.20 & 2 & 189.755 & 6 & 6 & 600.96 \\
\hline 26 & R109-25 & - & - & - & - & - & - & - & - \\
\hline 27 & R110-25 & - & - & - & - & - & - & - & - \\
\hline 28 & R111-25 & 879.85 & 4 & 385.20 & 2 & 174.648 & 7 & 6 & 559.85 \\
\hline 29 & R112-25 & - & - & - & - & - & 0 & 0 & 0.00 \\
\hline 30 & R201-25 & 552.19 & 3 & 402.19 & 0 & 0.000 & 0 & 3 & 402.19 \\
\hline 31 & R202-25 & 579.94 & 3 & 429.94 & 0 & 0.000 & 0 & 3 & 429.94 \\
\hline 32 & R203-25 & - & - & - & - & - & 0 & 0 & 0.00 \\
\hline 33 & R204-25 & 409.38 & 2 & 309.38 & 0 & 0.000 & 0 & 2 & 309.38 \\
\hline 34 & $\mathrm{R} 205-25$ & 553.97 & 3 & 403.97 & 0 & 0.000 & 0 & 3 & 403.97 \\
\hline 35 & R206-25 & 542.67 & 3 & 392.67 & 0 & 0.000 & 0 & 3 & 392.67 \\
\hline 36 & $\mathrm{R} 207-25$ & 440.55 & 2 & 340.55 & 0 & 0.000 & 0 & 2 & 340.55 \\
\hline 37 & R208-25 & 406.38 & 2 & 306.38 & 0 & 0.000 & 0 & 2 & 306.38 \\
\hline 38 & R209-25 & - & - & - & - & - & - & - & - \\
\hline 39 & $\mathrm{R} 210-25$ & 434.43 & 2 & 334.43 & 0 & 0.000 & 0 & 2 & 334.43 \\
\hline 40 & $\mathrm{R} 211-25$ & 468.92 & 2 & 368.92 & 0 & 0.000 & 0 & 2 & 368.92 \\
\hline 41 & $\mathrm{RC} 101-25$ & - & - & - & - & - & - & - & - \\
\hline 42 & RC102-25 & 1378.11 & 7 & 687.71 & 2 & 220.408 & 5 & 9 & 908.12 \\
\hline 43 & RC103-25 & 981.52 & 4 & 468.77 & 2 & 192.752 & 7 & 6 & 661.52 \\
\hline 44 & $\mathrm{RC} 104-25$ & - & - & - & - & - & - & - & - \\
\hline 45 & $\mathrm{RC} 105-25$ & 1243.32 & 6 & 604.86 & 2 & 218.459 & 6 & 8 & 823.32 \\
\hline 46 & $\mathrm{RC} 106-25$ & 1135.45 & 5 & 584.28 & 2 & 181.172 & 6 & 7 & 765.45 \\
\hline 47 & $\mathrm{RC} 107-25$ & - & - & - & - & - & - & - & - \\
\hline 48 & RC108-25 & 979.20 & 5 & 493.28 & 1 & 175.920 & 4 & 6 & 669.20 \\
\hline 49 & RC201-25 & 789.19 & 4 & 589.19 & 0 & 0.000 & 0 & 4 & 589.19 \\
\hline 50 & RC202-25 & 678.75 & 3 & 528.75 & 0 & 0.000 & 0 & 3 & 528.75 \\
\hline 51 & RC203-25 & 612.73 & 3 & 462.73 & 0 & 0.000 & 0 & 3 & 462.73 \\
\hline 52 & RC204-25 & 591.83 & 3 & 441.83 & 0 & 0.000 & 0 & 3 & 441.83 \\
\hline 53 & RC205-25 & 774.52 & 4 & 574.52 & 0 & 0.000 & 0 & 4 & 574.52 \\
\hline 54 & RC206-25 & 720.24 & 3 & 570.24 & 0 & 0.000 & 0 & 3 & 570.24 \\
\hline 55 & $\mathrm{RC} 207-25$ & 623.78 & 3 & 473.78 & 0 & 0.000 & 0 & 3 & 473.78 \\
\hline 56 & RC208-25 & 604.01 & 3 & 454.01 & 0 & 0.000 & 0 & 3 & 454.01 \\
\hline
\end{tabular}


Table B.2. Comparison of the proposed algorithms with results from CPLEX to small instances

\begin{tabular}{|c|c|c|c|c|c|c|c|c|c|c|c|}
\hline \multirow{2}{*}{ No. } & \multirow{2}{*}{ Inst. } & \multirow{2}{*}{$\begin{array}{l}\text { CPLEX } \\
\text { Cost }\end{array}$} & \multicolumn{3}{|l|}{ ALG-I } & \multicolumn{3}{|l|}{ ALG-II } & \multicolumn{3}{|l|}{ ALG-III } \\
\hline & & & Cost & $\mathrm{t}(\mathrm{s})$ & Gap & Cost & $\mathrm{t}(\mathrm{s})$ & Gap & Cost & $\mathrm{t}(\mathrm{s})$ & Gap \\
\hline 1 & C101-5 & 523.84 & 536.7859 & 0.01 & 0.02 & 523.835 & 0.03 & 0.00 & 523.835 & 0.02 & 0.00 \\
\hline 2 & C103-5 & 282.66 & 290.4764 & 0.01 & 0.03 & 282.6619 & 0.04 & 0.00 & 282.6619 & 0.04 & 0.00 \\
\hline 3 & C206-5 & 472.31 & 472.3065 & 0.01 & 0.00 & 472.3065 & 0.04 & 0.00 & 472.3065 & 0.02 & 0.00 \\
\hline 4 & C208-5 & 406.45 & 406.4544 & 0.01 & 0.00 & 406.4544 & 0.03 & 0.00 & 406.4544 & 0.01 & 0.00 \\
\hline 5 & R104-5 & 397.10 & 436.6159 & 0.02 & 0.10 & 400.7444 & 0.06 & 0.01 & 400.7444 & 0.03 & 0.01 \\
\hline 6 & R105-5 & 386.40 & 386.4031 & 0.01 & 0.00 & 386.4031 & 0.05 & 0.00 & 386.4031 & 0.04 & 0.00 \\
\hline 7 & $\mathrm{R} 202-5$ & 307.50 & 307.498 & 0.01 & 0.00 & 307.498 & 0.02 & 0.00 & 307.498 & 0.02 & 0.00 \\
\hline 8 & R203-5 & 506.42 & 567.8349 & 0.02 & 0.12 & 506.421 & 0.04 & 0.00 & 506.421 & 0.03 & 0.00 \\
\hline 9 & $\mathrm{RC} 105-5$ & 533.72 & 544.0631 & 0.04 & 0.02 & 533.715 & 0.10 & 0.00 & 533.715 & 0.13 & 0.00 \\
\hline 10 & RC108-5 & 585.99 & 648.3052 & 0.02 & 0.11 & 585.988 & 0.08 & 0.00 & 585.988 & 0.08 & 0.00 \\
\hline 11 & RC204-5 & 379.06 & 379.057 & 0.01 & 0.00 & 379.0569 & 0.04 & 0.00 & 379.0569 & 0.01 & 0.00 \\
\hline 12 & RC208-5 & 386.61 & 408.0865 & 0.02 & 0.06 & 386.608 & 0.12 & 0.00 & 386.608 & 0.07 & 0.00 \\
\hline 13 & C101-10 & 719.85 & 785.5532 & 0.01 & 0.09 & 719.848 & 0.13 & 0.00 & 719.848 & 0.03 & 0.00 \\
\hline 14 & C104-10 & 442.87 & 442.8674 & 0.05 & 0.00 & 442.8674 & 0.40 & 0.00 & 442.8674 & 0.13 & 0.00 \\
\hline 15 & C202-10 & 534.76 & 534.755 & 0.03 & 0.00 & 534.755 & 0.20 & 0.00 & 534.755 & 0.13 & 0.00 \\
\hline 16 & C205-10 & 518.49 & 530.1478 & 0.07 & 0.02 & 530.1478 & 0.32 & 0.02 & 530.1478 & 0.21 & 0.02 \\
\hline 17 & R102-10 & 564.57 & 612.6751 & 0.03 & 0.09 & 564.572 & 0.12 & 0.00 & 564.572 & 0.11 & 0.00 \\
\hline 18 & R103-10 & 379.81 & 386.4048 & 0.03 & 0.02 & 379.8145 & 0.17 & 0.00 & 379.8145 & 0.10 & 0.00 \\
\hline 19 & R201-10 & 462.70 & 462.697 & 0.09 & 0.00 & 462.697 & 0.46 & 0.00 & 462.697 & 0.35 & 0.00 \\
\hline 20 & R203-10 & 475.95 & 559.3998 & 0.16 & 0.18 & 475.949 & 0.47 & 0.00 & 475.949 & 0.35 & 0.00 \\
\hline 21 & RC102-10 & 852.75 & 938.0435 & 0.06 & 0.10 & 852.752 & 0.12 & 0.00 & 852.752 & 0.12 & 0.00 \\
\hline 22 & RC108-10 & 757.02 & 763.613 & 0.06 & 0.01 & 825.0483 & 0.20 & 0.09 & 763.613 & 0.20 & 0.01 \\
\hline 23 & RC201-10 & 577.54 & 577.535 & 0.06 & 0.00 & 577.535 & 0.51 & 0.00 & 577.535 & 0.17 & 0.00 \\
\hline 24 & RC205-10 & 723.13 & 822.1292 & 0.05 & 0.14 & 723.125 & 0.17 & 0.00 & 723.125 & 0.12 & 0.00 \\
\hline 25 & C103-15 & 652.03 & 671.3654 & 0.18 & 0.03 & 654.0996 & 0.48 & 0.00 & 656.387 & 0.50 & 0.01 \\
\hline 26 & C106-15 & 603.04 & 603.042 & 0.12 & 0.00 & 603.042 & 0.22 & 0.00 & 603.042 & 0.32 & 0.00 \\
\hline 27 & C202-15 & 689.64 & 689.64 & 0.10 & 0.00 & 689.64 & 0.53 & 0.00 & 689.64 & 0.49 & 0.00 \\
\hline 28 & C208-15 & 582.12 & 582.117 & 0.36 & 0.00 & 582.117 & 0.94 & 0.00 & 582.117 & 1.35 & 0.00 \\
\hline 29 & R102-15 & 645.18 & 728.0406 & 0.11 & 0.13 & 645.181 & 0.47 & 0.00 & 645.181 & 0.46 & 0.00 \\
\hline 30 & R105-15 & 803.36 & 814.2376 & 0.08 & 0.01 & 813.9432 & 0.52 & 0.01 & 814.2376 & 0.16 & 0.01 \\
\hline 31 & R202-15 & 741.52 & 805.9605 & 0.21 & 0.09 & 791.8202 & 0.76 & 0.07 & 795.6354 & 1.10 & 0.07 \\
\hline 32 & R209-15 & 562.96 & 562.958 & 2.79 & 0.00 & 562.958 & 7.63 & 0.00 & 562.958 & 4.77 & 0.00 \\
\hline 33 & RC103-15 & 975.68 & 926.3873 & 0.17 & $-0.05^{*}$ & 902.3177 & 0.33 & -0.08 & 920.0647 & 0.36 & -0.06 \\
\hline 34 & $\mathrm{RC} 108-15$ & 783.27 & 783.27 & 0.18 & 0.00 & 783.27 & 1.17 & 0.00 & 783.27 & 0.54 & 0.00 \\
\hline 35 & RC202-15 & 660.09 & 714.1996 & 0.61 & 0.08 & 660.088 & 2.77 & 0.00 & 660.088 & 3.61 & 0.00 \\
\hline 36 & RC204-15 & 602.31 & 602.309 & 8.00 & 0.00 & 602.309 & 13.62 & 0.00 & 602.309 & 18.18 & 0.00 \\
\hline
\end{tabular}


Table B.3. Comparison of the proposed algorithms with results from CPLEX to instances with 25 customers

\begin{tabular}{|c|c|c|c|c|c|c|c|c|c|c|c|}
\hline \multirow{2}{*}{ No. } & \multirow{2}{*}{ Inst. } & \multirow{2}{*}{$\begin{array}{l}\text { CPLEX } \\
\text { Cost }\end{array}$} & \multicolumn{3}{|l|}{ ALG-I } & \multicolumn{3}{|l|}{ ALG-II } & \multicolumn{3}{|l|}{ ALG-III } \\
\hline & & & Cost & $\mathrm{t}(\mathrm{s})$ & Gap & Cost & $\mathrm{t}(\mathrm{s})$ & Gap & Cost & $\mathrm{t}(\mathrm{s})$ & Gap \\
\hline 1 & C101-25 & 1175.42 & 1204.37 & 23.64 & 0.02 & 1175.42 & 70.68 & 0.00 & 1175.42 & 88.76 & 0.00 \\
\hline 2 & $\mathrm{C} 102-25$ & $1045.82^{*}$ & 1091.12 & 28.35 & 0.04 & 1046.11 & 84.73 & 0.00 & 1075.20 & 33.24 & 0.03 \\
\hline 3 & C103-25 & - & 754.32 & 35.21 & - & 721.61 & 163.69 & - & 723.54 & 44.43 & - \\
\hline 4 & C104-25 & 859.85 & 902.37 & 53.16 & 0.05 & 834.21 & 58.14 & -0.03 & 861.42 & 50.47 & 0.00 \\
\hline 5 & $\mathrm{C} 105-25$ & 1032.38 & 1079.75 & 29.04 & 0.05 & 1048.01 & 106.15 & 0.02 & 1048.01 & 151.39 & 0.02 \\
\hline 6 & C106-25 & 1090.57 & 1163.31 & 28.56 & 0.07 & 1090.57 & 50.32 & 0.00 & 1090.57 & 55.59 & 0.00 \\
\hline 7 & C107-25 & 960.44 & 1014.13 & 46.44 & 0.06 & 960.44 & 27.40 & 0.00 & 960.44 & 24.68 & 0.00 \\
\hline 8 & C108-25 & - & 1009.42 & 36.99 & - & 897.02 & 121.54 & - & 897.02 & 37.84 & - \\
\hline 9 & C109-25 & 875.38 & 918.36 & 44.51 & 0.05 & 875.38 & 76.21 & 0.00 & 875.38 & 41.36 & 0.00 \\
\hline 10 & C201-25 & 687.80 & 687.80 & 15.89 & 0.00 & 687.80 & 79.82 & 0.00 & 687.80 & 87.09 & 0.00 \\
\hline 11 & $\mathrm{C} 202-25$ & 687.39 & 687.39 & 35.29 & 0.00 & 687.39 & 109.87 & 0.00 & 687.39 & 47.61 & 0.00 \\
\hline 12 & C203-25 & 738.61 & 713.75 & 15.12 & -0.03 & 713.75 & 62.02 & -0.03 & 713.75 & 30.22 & -0.03 \\
\hline 13 & C204-25 & 557.76 & 524.89 & 15.00 & -0.06 & 508.88 & 43.93 & -0.09 & 508.88 & 54.12 & -0.09 \\
\hline 14 & C205-25 & 636.22 & 680.32 & 26.15 & 0.07 & 636.22 & 56.03 & 0.00 & 636.22 & 35.13 & 0.00 \\
\hline 15 & C206-25 & 680.04 & 680.04 & 16.43 & 0.00 & 680.04 & 84.69 & 0.00 & 680.04 & 70.51 & 0.00 \\
\hline 16 & $\mathrm{C} 207-25$ & 667.74 & 708.02 & 35.01 & 0.06 & 667.74 & 21.46 & 0.00 & 667.74 & 28.44 & 0.00 \\
\hline 17 & C208-25 & - & 689.97 & 12.19 & - & 689.97 & 27.19 & - & 689.97 & 51.27 & - \\
\hline 18 & R101-25 & 1330.84 & 1330.84 & 39.06 & 0.00 & 1330.84 & 85.95 & 0.00 & 1330.84 & 155.09 & 0.00 \\
\hline 19 & $\mathrm{R} 102-25$ & 1207.84 & 1223.45 & 115.07 & 0.01 & 1223.45 & 135.41 & 0.01 & 1207.84 & 84.10 & 0.00 \\
\hline 20 & R103-25 & - & 935.72 & 74.09 & - & 912.18 & 79.64 & - & 929.94 & 65.28 & - \\
\hline 21 & R104-25 & 739.50 & 741.15 & 28.76 & 0.00 & 730.15 & 96.85 & -0.01 & 730.15 & 84.31 & -0.01 \\
\hline 22 & $\mathrm{R} 105-25$ & 1144.43 & 1175.26 & 34.15 & 0.03 & 1175.26 & 46.51 & 0.03 & 1175.26 & 35.29 & 0.03 \\
\hline 23 & R106-25 & 996.51 & 1026.37 & 28.86 & 0.03 & 996.51 & 110.77 & 0.00 & 1006.08 & 151.87 & 0.01 \\
\hline 24 & R107-25 & 892.55 & 936.92 & 62.19 & 0.05 & 892.55 & 68.74 & 0.00 & 895.02 & 68.06 & 0.00 \\
\hline 25 & R108-25 & 920.96 & 923.12 & 54.31 & 0.00 & 929.35 & 36.31 & 0.01 & 961.31 & 90.89 & 0.04 \\
\hline 26 & R109-25 & - & 992.51 & 31.52 & - & 1003.58 & 75.58 & - & 1027.19 & 36.83 & - \\
\hline 27 & $\mathrm{R} 110-25$ & - & 944.93 & 32.40 & - & 849.96 & 32.58 & - & 914.64 & 74.89 & - \\
\hline 28 & R111-25 & 879.85 & 879.85 & 43.25 & 0.00 & 879.85 & 163.00 & 0.00 & 879.85 & 164.10 & 0.00 \\
\hline 29 & R112-25 & - & 825.06 & 28.54 & - & 825.06 & 119.74 & - & 840.81 & 71.74 & - \\
\hline 30 & $\mathrm{R} 201-25$ & 552.19 & 552.19 & 25.26 & 0.00 & 552.19 & 53.12 & 0.00 & 552.19 & 37.05 & 0.00 \\
\hline 31 & $\mathrm{R} 202-25$ & 579.94 & 579.94 & 18.57 & 0.00 & 579.94 & 14.20 & 0.00 & 579.94 & 45.56 & 0.00 \\
\hline 32 & R203-25 & - & 673.47 & 28.39 & - & 660.19 & 22.84 & - & 668.55 & 56.72 & - \\
\hline 33 & R204-25 & 409.38 & 443.35 & 18.91 & 0.08 & 409.38 & 82.27 & 0.00 & 409.38 & 36.16 & 0.00 \\
\hline 34 & $\mathrm{R} 205-25$ & 553.97 & 594.19 & 32.54 & 0.07 & 594.19 & 120.10 & 0.07 & 553.97 & 34.15 & 0.00 \\
\hline 35 & R206-25 & 542.67 & 542.67 & 18.35 & 0.00 & 542.67 & 42.87 & 0.00 & 542.67 & 30.39 & 0.00 \\
\hline 36 & R207-25 & 440.55 & 440.55 & 21.56 & 0.00 & 440.55 & 32.99 & 0.00 & 440.55 & 46.75 & 0.00 \\
\hline 37 & R208-25 & 406.38 & 406.38 & 23.81 & 0.00 & 406.38 & 25.07 & 0.00 & 406.38 & 13.65 & 0.00 \\
\hline 38 & R209-25 & - & 601.28 & 17.56 & - & 584.58 & 35.86 & - & 589.62 & 23.04 & - \\
\hline 39 & $\mathrm{R} 210-25$ & 434.43 & 434.43 & 31.99 & 0.00 & 434.43 & 72.13 & 0.00 & 434.43 & 26.14 & 0.00 \\
\hline 40 & R211-25 & 468.92 & 468.92 & 15.53 & 0.00 & 468.92 & 36.15 & 0.00 & 468.92 & 14.35 & 0.00 \\
\hline 41 & $\mathrm{RC} 101-25$ & - & 1469.57 & 32.78 & - & 1415.15 & 204.27 & - & 1415.15 & 170.85 & - \\
\hline 42 & $\mathrm{RC} 102-25$ & 1378.11 & 1378.11 & 76.98 & 0.00 & 1381.01 & 94.28 & 0.00 & 1378.11 & 140.46 & 0.00 \\
\hline 43 & RC103-25 & 981.52 & 1003.49 & 35.17 & 0.02 & 974.43 & 62.12 & -0.01 & 974.43 & 118.33 & -0.01 \\
\hline 44 & RC104-25 & - & 1131.43 & 48.90 & - & 1131.43 & 69.54 & - & 1155.67 & 53.67 & - \\
\hline 45 & $\mathrm{RC} 105-25$ & 1243.32 & 1310.32 & 60.77 & 0.05 & 1243.32 & 126.06 & 0.00 & 1310.32 & 64.81 & 0.05 \\
\hline 46 & $\mathrm{RC} 106-25$ & 1135.45 & 1198.74 & 97.60 & 0.06 & 1138.23 & 133.82 & 0.00 & 1138.23 & 105.41 & 0.00 \\
\hline 47 & $\mathrm{RC} 107-25$ & - & 1111.05 & 44.84 & - & 1050.02 & 59.47 & - & 1050.02 & 100.44 & - \\
\hline 48 & $\mathrm{RC} 108-25$ & 979.20 & 966.96 & 27.83 & -0.01 & 962.40 & 57.22 & -0.02 & 962.40 & 136.99 & -0.02 \\
\hline 49 & RC201-25 & 789.19 & 789.19 & 50.69 & 0.00 & 789.19 & 78.04 & 0.00 & 789.19 & 95.37 & 0.00 \\
\hline 50 & RC202-25 & 678.75 & 678.75 & 30.26 & 0.00 & 678.75 & 139.63 & 0.00 & 678.75 & 89.67 & 0.00 \\
\hline 51 & RC203-25 & 612.73 & 612.73 & 12.00 & 0.00 & 612.73 & 27.75 & 0.00 & 612.73 & 104.11 & 0.00 \\
\hline 52 & RC204-25 & 591.83 & 647.63 & 14.61 & 0.09 & 637.32 & 96.51 & 0.08 & 637.32 & 26.76 & 0.08 \\
\hline 53 & $\mathrm{RC} 205-25$ & 774.52 & 822.71 & 21.12 & 0.06 & 774.52 & 65.68 & 0.00 & 822.71 & 62.32 & 0.06 \\
\hline 54 & RC206-25 & 720.24 & 781.05 & 25.65 & 0.08 & 720.24 & 81.90 & 0.00 & 720.24 & 33.76 & 0.00 \\
\hline 55 & $\mathrm{RC} 207-25$ & 623.78 & 623.78 & 24.07 & 0.00 & 623.78 & 27.43 & 0.00 & 623.78 & 31.06 & 0.00 \\
\hline 56 & RC208-25 & 604.01 & 604.01 & 10.63 & 0.00 & 604.01 & 15.33 & 0.00 & 604.01 & 62.28 & 0.00 \\
\hline
\end{tabular}




\section{References}

Alice/Ertrac (2015). Urban freight research roadmap. Brussels. Retrieved from: https://www.ertrac.org/uploads/documentsearch/id36/ERTRAC_Alice_Urban_Freight.pdf.

Allen, J., Browne, M., \& Piecyk, M. (2017). Assessing the European Commission's target of essentially CO2-free city logistics in urban centres by 2030. Retrieved from: http://www.citylab-project.eu/deliverables/D2_4.pdf.

Anderluh, A., Hemmelmayr, V. C., \& Nolz, P. C. (2017). Synchronizing vans and cargo bikes in a city distribution network. Central European Journal of Operations Research, 25(2), 345-376.

Androutsopoulos, K. N., \& Zografos, K. G. (2017). An integrated modelling approach for the bicriterion vehicle routing and scheduling problem with environmental considerations. Transportation Research Part C: Emerging Technologies, 82, 180-209.

Basso, R., Kulcsár, B., Egardt, B., Lindroth, P., \& Sanchez-Diaz, I. (2019). Energy consumption estimation integrated into the Electric Vehicle Routing Problem. Transportation Research Part D: Transport and Environment 69, $141-167$.

Bektaş, T., Ehmke, J. F., Psaraftis, H. N., \& Puchinger, J. (2019). The Role of Operational Research in Green Freight Transportation. European Journal of Operational Research, 274(3), 807-823.

Bektas, T., \& Laporte, G. (2011). The Pollution-Routing Problem. Transportation Research Part B-Methodological, $45(8), 1232-1250$

Bent, R., \& Van Hentenryck, P. (2004). A two-stage hybrid local search for the vehicle routing problem with time windows. Transportation Science, 38(4), 515-530.

Bruglieri, M., Pezzella, F., Pisacane, O., \& Suraci, S. (2015). A variable neighborhood search branching for the electric vehicle routing problem with time windows. Electronic Notes in Discrete Mathematics, 47, $221-228$. 4069.

Carlsson, J. G., \& Song, S. (2018). Coordinated logistics with a truck and a drone. Management Science, 64(9), 4052-

Committee on Climate Change. (2010). The Fourth Carbon Budget - reducing emissions through the 2020s. Retrieved from: https://www.theccc.org.uk/wp-content/uploads/2010/09/CCC-4th-Budget-Book_with-hypers.pdf.

Conrad, R. G., \& Figliozzi, M. A. (2011, May). The recharging vehicle routing problem. In Proceedings of the 2011 industrial engineering research conference (p. 8). IISE Norcross, GA.

Crainic, T. G., Ricciardi, N., \& Storchi, G. (2009). Models for Evaluating and Planning City Logistics Systems. Transportation Science, 43(4), 432-454.

Davis, B. A., \& Figliozzi, M. A. (2013). A methodology to evaluate the competitiveness of electric delivery trucks. Transportation Research Part E: Logistics and Transportation Review, 49, 8-23.

Demir, E., Bektas, T., \& Laporte, G. (2014). The bi-objective Pollution-Routing Problem. European Journal of Operational Research, 232(3), 464-478.

Desaulniers, G., Errico, F., Irnich, S., \& Schneider, M. (2016). Exact Algorithms for Electric Vehicle-Routing Problems with Time Windows. Operations Research, 64(6), 1388-1405.

Drexl, M. (2012). Synchronization in Vehicle Routing-A Survey of VRPs with Multiple Synchronization Constraints. Transportation Science, 46(3), 297-316.

Erdogan, S., \& Miller-Hooks, E. (2012). A Green Vehicle Routing Problem. Transportation Research Part ELogistics and Transportation Review, 48(1), 100-114.

European Comission. Directorate-General for Mobility and Transport. (2011). White Paper on Transport: Roadmap to a Single European Transport Area: Towards a Competitive and Resource-efficient Transport System. Publications Office of the European Union.

Felipe, A., Ortuno, M. T., Righini, G., \& Tirado, G. (2014). A heuristic approach for the green vehicle routing problem with multiple technologies and partial recharges. Transportation Research Part E-Logistics and Transportation Review, 71, 111-128.

Feng, W., \& Figliozzi, M. (2013). An economic and technological analysis of the key factors affecting the competitiveness of electric commercial vehicles: A case study from the USA market. Transportation Research Part C: Emerging Technologies, 26, 135-145. 
Froger, A., Mendoza, J. E., Jabali, O., \& Laporte, G. (2019). Improved formulations and algorithmic components for the electric vehicle routing problem with nonlinear charging functions. Computers \& Operations Research, 104, $256-294$.

Franceschetti, A., Honhon, D., Van Woensel, T., Bektas, T., \& Laporte, G. (2013). The time-dependent pollutionrouting problem. Transportation Research Part B-Methodological, 56, 265-293.

Gao, X. J., Zhao, J. L., Shang, W. Z., Wang, T. B., Wang, X. (2 May 2012) A Mobile Electric Vehicle Battery Swapping Van for Emergency. CN 201120341622.2, 2.

Goeke, D., \& Schneider, M. (2015). Routing a mixed fleet of electric and conventional vehicles. European Journal of Operational Research, 245(1), 81-99.

González-Rodríguez, P. L., Canca, D., Andrade-Pineda, J. L., Calle, M., \& Leon-Blanco, J. M. (2020). Truck-drone team logistics: A heuristic approach to multi-drop route planning. Transportation Research Part C: Emerging Technologies, $114,657-680$.

Grangier, P., Gendreau, M., Lehuede, F., \& Rousseau, L. M. (2016). An adaptive large neighborhood search for the two-echelon multiple-trip vehicle routing problem with satellite synchronization. European Journal of Operational Research, 254(1), 80-91.

Hiermann, G., Puchinger, J., Ropke, S., \& Hartl, R. F. (2016). The Electric Fleet Size and Mix Vehicle Routing Problem with Time Windows and Recharging Stations. European Journal of Operational Research, 252(3), $995-1018$.

Hof, J., Schneider, M., \& Goeke, D. (2017). Solving the battery swap station location-routing problem with capacitated electric vehicles using an AVNS algorithm for vehicle-routing problems with intermediate stops. Transportation Research Part B-Methodological, 97, 102-112.

Karak, A., \& Abdelghany, K. (2019). The hybrid vehicle-drone routing problem for pick-up and delivery services. Transportation Research Part C: Emerging Technologies, 102, 427-449.

Keskin, M., \& Çatay, B. (2016). Partial recharge strategies for the electric vehicle routing problem with time windows. Transportation Research Part C-Emerging Technologies, 65, 111-127.

Kitjacharoenchai, P., Min, B. C., \& Lee, S. (2020). Two echelon vehicle routing problem with drones in last mile delivery. International Journal of Production Economics, 225, 107598.

Koc, C., Bektas, T., Jabali, O., \& Laporte, G. (2014). The fleet size and mix pollution-routing problem. Transportation Research Part B-Methodological, 70, 239-254.

Kullman, N., Goodson, J., Mendoza, J.E., (2018). Dynamic electric vehicle routing with mid-route recharging and uncertain availability. ODYSSEUS 2018 - Seventh International Workshop on Freight Transportation and Logistics. Cagliari, Italy.

Lee, D. Y., Thomas, V. M., \& Brown, M. A. (2013). Electric urban delivery trucks: Energy use, greenhouse gas emissions, and cost-effectiveness. Environmental science \& technology, 47(14), 8022-8030.

Lu, G. J., \& Zhou, Y. P. (16 October 2013). A Electric Vehicle with Bottom Lateral Linkage Battery Swapping and Its Battery Swapping Devices. CN 201310164242.X.

Macrina, G., Pugliese, L. D. P., Guerriero, F., \& Laporte, G. (2019). The green mixed fleet vehicle routing problem with partial battery recharging and time windows. Computers \& Operations Research 101, 183-199.

MDS Transmodal. (2012). DG MOVE European Commission: Study on urban freight transport. Final report. Retrieved from: https:/ec.europa.eu/transport/sites/transport/files/themes/urban/studies/doc/2012-04-urban-freighttransport.pdf

Montoya, A., Gueret, C., Mendoza, J. E., \& Villegas, J. G. (2017). The electric vehicle routing problem with nonlinear charging function. Transportation Research Part B-Methodological, 103, 87-110.

Murray, C. C., \& Chu, A. G. (2015). The flying sidekick traveling salesman problem: Optimization of drone-assisted parcel delivery. Transportation Research Part C: Emerging Technologies, 54, 86-109.

Pelletier, S., Jabali, O., \& Laporte, G. (2016). Goods Distribution with Electric Vehicles: Review and Research Perspectives. Transportation Science, 50(1), 3-22.

Poikonen, S., Wang, X., \& Golden, B. (2017). The vehicle routing problem with drones: Extended models and connections. Networks, 70(1), 34-43.

Raeesi, R., \& O’Sullivan, M. J. (2014). Eco-logistics: environmental and economic implications of alternative fuel vehicle routing problem. International Journal of Business Performance and Supply Chain Modelling, 6(3-4), $276-297$. 
Raeesi, R., \& Zografos, K. G. (2019). The multi-objective Steiner pollution-routing problem on congested urban road networks. Transportation Research Part B: Methodological, 122, 457-485.

Ritchie, Hanna, \& Roser, M. (2018). Urbanization. Published online at OurWorldInData.org Retrieved from: https://ourworldindata.org/urbanization.

Ropke, S., \& Pisinger, D. (2006). An adaptive large neighborhood search heuristic for the pickup and delivery problem with time windows. Transportation Science, 40(4), 455-472.

Salimifard, K., \& Raeesi, R. (2014). A green routing problem: optimising CO2 emissions and costs from a bi-fuel vehicle fleet. International Journal of Advanced Operations Management, 6(1), 27-57.

Schermer, D., Moeini, M., \& Wendt, O. (2019). A matheuristic for the vehicle routing problem with drones and its variants. Transportation Research Part C: Emerging Technologies, 106, 166-204.

Schiffer, M., Stütz, S., \& Walther, G. (2017). Are ECVs breaking even? - Competitiveness of electric commercial vehicles in retail logistics. Technical Report G-2017-47. RWTH Aachen University. Aachen. URL: https://publications.rwth-aachen.de/record/691766.

Schiffer, M., \& Walther, G. (2017). The electric location routing problem with time windows and partial recharging. European Journal of Operational Research, 260(3), 995-1013.

Schneider, M., Stenger, A., \& Goeke, D. (2014). The Electric Vehicle-Routing Problem with Time Windows and Recharging Stations. Transportation Science, 48(4), 500-520.

Shao, S. J., Guo, S. Y., \& Qiu, X. S. (2017). A Mobile Battery Swapping Service for Electric Vehicles Based on a Battery Swapping Van. Energies, 10(10), 1667.

Shaw, P. (1998). Using constraint programming and local search methods to solve vehicle routing problems. Principles and Practice of Constraint Programming - Cp98, 1520, 417-431.

Solomon, M. M. (1987). Algorithms for the vehicle routing and scheduling problems with time window constraints. Operations research, 35(2), 254-265.

Sweda, T. M., Dolinskaya, I. S., \& Klabjan, D. (2017). Adaptive routing and recharging policies for electric vehicles. Transportation Science, 51(4), 1326-1348.

Worley, O., Klabjan, D., \& Sweda, T. M. (2012). Simultaneous vehicle routing and charging station siting for commercial electric vehicles. In 2012 IEEE International Electric Vehicle Conference (pp. 1-3), Greenville, NC.

Yang, J., \& Sun, H. (2015). Battery swap station location-routing problem with capacitated electric vehicles. Computers \& Operations Research, 55, 217-232. 